JOURNAL OF THE

AMERICAN MATHEMATICAL SOCIETY

Volume 18, Number 1, Pages 121-156

S 0894-0347(04)00469-2

Article electronically published on September 16, 2004

\title{
ANALYSIS OF THE HETEROGENEOUS MULTISCALE METHOD FOR ELLIPTIC HOMOGENIZATION PROBLEMS
}

\author{
WEINAN E, PINGBING MING, AND PINGWEN ZHANG
}

\section{Contents}

1. Introduction and main results

1.1. General methodology $\quad 122$

1.2. Heterogeneous multiscale method $\quad 122$

1.3. Main results 125

1.4. Recovering the microstructural information $\quad 126$

1.5. Some technical background 127

2. Generalities 129

3. Estimating $e(\mathrm{HMM}) \quad 132$

4. Reconstruction and compression $\quad 136$

4.1. Reconstruction procedure 136

4.2. Compression operator 138

5. Nonlinear homogenization problems $\quad 139$

5.1. Algorithms and main results $\quad 139$

5.2. Estimating $e(\mathrm{HMM}) \quad 143$

Appendix A. Estimating $e(\mathrm{HMM})$ for problems with random coefficients 149

\begin{tabular}{ll} 
References & 154 \\
\hline
\end{tabular}

Received by the editors January 2, 2003

2000 Mathematics Subject Classification. Primary 65N30, 74Q05; Secondary 74Q15, 65C30.

Key words and phrases. Heterogeneous multiscale method, homogenization problems.

The work of the first author was partially supported by ONR grant N00014-01-1-0674 and the National Natural Science Foundation of China through a Class B Award for Distinguished Young Scholars 10128102.

The work of the second author was partially supported by the Special Funds for the Major State Basic Research Projects G1999032804 and was also supported by the National Natural Science Foundation of China 10201033.

The work of the third author was partially supported by the Special Funds for the Major State Research Projects G1999032804 and the National Natural Science Foundation of China for Distinguished Young Scholars 10225103.

We thank Bjorn Engquist for inspiring discussions on the topic studied here.

(C)2004 American Mathematical Society
Reverts to public domain 28 years from publication 


\section{IntRoduCtion AND MAIN RESULtS}

1.1. General methodology. Consider the classical elliptic problem

$$
\left\{\begin{aligned}
-\operatorname{div}\left(a^{\varepsilon}(x) \nabla u^{\varepsilon}(x)\right) & =f(x) & & x \in D \subset \mathbb{R}^{d}, \\
u^{\varepsilon}(x) & =0 & & x \in \partial D .
\end{aligned}\right.
$$

Here $\varepsilon$ is a small parameter that signifies explicitly the multiscale nature of the coefficient $a^{\varepsilon}(x)$. Several classical multiscale methodologies have been developed for the numerical solution of this elliptic problem, the most well known among which is the multigrid technique [8]. These classical multiscale methods are designed to resolve the details of the fine scale problem (1.1) and are applicable for general problems, i.e., no special assumptions are required for the coefficient $a^{\varepsilon}(x)$. In contrast modern multiscale methods are designed specifically for recovering partial information about $u^{\varepsilon}$ at a sublinear cost, i.e., the total cost grows sublinearly with the cost of solving the fine scale problem [18]. This is only possible by exploring the special features that $a^{\varepsilon}(x)$ might have, such as scale separation. The simplest example is when

$$
a^{\varepsilon}(x)=a\left(x, \frac{x}{\varepsilon}\right),
$$

where $a(x, y)$ can either be periodic in $y$, in which case we assume the period to be $I=[-1 / 2,1 / 2]^{d}$, or random but stationary under shifts in $y$, for each fixed $x \in D$. In both cases, it has been shown that [5, 36]

$$
\left\|u^{\varepsilon}(x)-U(x)\right\|_{L^{2}(D)} \rightarrow 0,
$$

where $U(x)$ is the solution of a homogenized equation:

$$
\left\{\begin{aligned}
-\operatorname{div}(\mathcal{A}(x) \nabla U(x)) & =f(x) & & x \in D, \\
U(x) & =0 & & x \in \partial D .
\end{aligned}\right.
$$

The homogenized coefficient $\mathcal{A}(x)$ can be obtained from the solutions of the socalled cell problem. In general, there are no explicit formulas for $\mathcal{A}(x)$, except in one dimension.

Several numerical methods have been developed to deal specifically with the case when $a(x, y)$ is periodic in $y$. References [3, 4, 7] propose to solve the homogenized equations as well as the equations for the correctors. Schwab et al. [29, 38] use multiscale test functions of the form $\varphi(x, x / \varepsilon)$ where $\varphi(x, y)$ is periodic in $y$ to extract the leading order behavior of $u^{\varepsilon}(x)$, extending an idea that was used analytically in the work of [2, 15, 34, 44] for the homogenization problems. These methods have the feature that their cost is independent of $\varepsilon$, hence sublinear as $\varepsilon \rightarrow 0$, but so far they are restricted to the periodic homogenization problem. An alternative proposal for more general problems but with much higher cost is found in [20, 25].

1.2. Heterogeneous multiscale method. HMM [16, 17, 18] is a general methodology for designing sublinear algorithms by exploiting scale separation and other special features of the problem. It consists of two components: selection of a macroscopic solver and estimating the missing macroscale data by solving locally the fine scale problem.

For (1.1) the macroscopic solver can be chosen as a conventional $\mathcal{P}_{k}$ finite element method on a triangulation $\mathcal{T}_{H}$ of element size $H$ which should resolve the macroscale features of $a^{\varepsilon}(x)$. The missing data is the effective stiffness matrix at 
this scale. This stiffness matrix can be estimated as follows. Assuming that the effective coefficient at this scale is $\mathcal{A}_{H}(x)$, if we knew $\mathcal{A}_{H}(x)$ explicitly, we could have evaluated the quadratic form

$$
\int_{D} \nabla V(x) \cdot \mathcal{A}_{H}(x) \nabla V(x) d x
$$

by numerical quadrature: For any $V \in X_{H}$, the finite element space,

$$
A_{H}(V, V) \simeq \sum_{K \in \mathcal{T}_{H}}|K| \sum_{x_{\ell} \in K} \omega_{\ell}\left(\nabla V \cdot \mathcal{A}_{H} \nabla V\right)\left(x_{\ell}\right),
$$

where $\left\{x_{\ell}\right\}$ and $\left\{\omega_{\ell}\right\}$ are the quadrature points and weights in $K,|K|$ is the volume of $K$. In the absence of explicit knowledge of $\mathcal{A}_{H}(x)$, we approximate $\left(\nabla V \cdot \mathcal{A}_{H} \nabla V\right)\left(x_{\ell}\right)$ by solving the problem:

$$
\left\{\begin{aligned}
-\operatorname{div}\left(a^{\varepsilon}(x) \nabla v_{\ell}^{\varepsilon}(x)\right) & =0 & & x \in I_{\delta}\left(x_{\ell}\right), \\
v_{\ell}^{\varepsilon}(x) & =V_{\ell}(x) & & x \in \partial I_{\delta}\left(x_{\ell}\right),
\end{aligned}\right.
$$

where $I_{\delta}\left(x_{\ell}\right)$ is a cube of size $\delta$ centered at $x_{\ell}$, and $V_{\ell}$ is the linear approximation of $V$ at $x_{\ell}$. We then let

$$
\left(\nabla V \cdot \mathcal{A}_{H} \nabla V\right)\left(x_{\ell}\right) \simeq \frac{1}{\delta^{d}} \int_{I_{\delta}\left(x_{\ell}\right)} \nabla v_{\ell}^{\varepsilon}(x) \cdot a^{\varepsilon}(x) \nabla v_{\ell}^{\varepsilon}(x) d x .
$$

(1.5) and (1.7) together give the needed approximate stiffness matrix at the scale $H$. For convenience, we will define the corresponding bilinear form: For any $V, W \in X_{H}$

$$
A_{H}(V, W):=\sum_{K \in \mathcal{T}_{H}} \frac{|K|}{\delta^{d}} \sum_{x_{\ell} \in K} \omega_{\ell} \int_{I_{\delta}\left(x_{\ell}\right)} \nabla v_{\ell}^{\varepsilon}(x) \cdot a^{\varepsilon}(x) \nabla w_{\ell}^{\varepsilon}(x) d x,
$$

where $w_{\ell}^{\varepsilon}$ is defined for $W \in X_{H}$ in the same way that $v_{\ell}^{\varepsilon}$ in (1.6) was defined for $V$.

In order to reduce the effect of the imposed boundary condition on $\partial I_{\delta}\left(x_{\ell}\right)$, we may replace (1.7) by

$$
\left(\nabla V \cdot \mathcal{A}_{H} \nabla V\right)\left(x_{\ell}\right) \simeq \frac{1}{\left(\delta^{\prime}\right)^{d}} \int_{I_{\delta^{\prime}}\left(x_{\ell}\right)} \nabla v_{\ell}^{\varepsilon}(x) \cdot a^{\varepsilon}(x) \nabla v_{\ell}^{\varepsilon}(x) d x,
$$

where $\delta^{\prime}<\delta$. For example, we may choose $\delta^{\prime}=\delta / 2$. In (1.6), we used the Dirichlet boundary condition. Other boundary conditions are possible, such as Neumann and periodic boundary conditions. In the case when $a^{\varepsilon}(x)=a(x, x / \varepsilon)$ and $a(x, y)$ is periodic in $y$, one can take $I_{\delta}\left(x_{\ell}\right)$ to be $x_{\ell}+\varepsilon I$, i.e., $\delta=\varepsilon$ and use the boundary condition that $v_{\ell}^{\varepsilon}(x)-V_{\ell}(x)$ is periodic on $I_{\delta}$.

So far the algorithm is completely general. The savings compared with solving the full fine scale problem comes from the fact that we can choose $I_{\delta}\left(x_{\ell}\right)$ to be smaller than $K$. The size of $I_{\delta}\left(x_{\ell}\right)$ is determined by many factors, including the accuracy and cost requirement, the degree of scale separation, and the microstructure in $a^{\varepsilon}(x)$. One purpose for the error estimates that we present below is to give guidelines on how to select $I_{\delta}\left(x_{\ell}\right)$. As mentioned already, if $a^{\varepsilon}(x)=a(x, x / \varepsilon)$ and $a(x, y)$ is periodic in $y$, we can simply choose $I_{\delta}\left(x_{\ell}\right)$ to be $x_{\ell}+\varepsilon I$, i.e., $\delta=\varepsilon$. If $a(x, y)$ is random, then $\delta$ should be a few times larger than the local correlation 
length of $a^{\varepsilon}$. In the former case, the total cost is independent of $\varepsilon$. In the latter case, the total cost depends only weakly on $\varepsilon$ (see [31]).

The final problem is to solve

$$
\min _{V \in X_{H}} \frac{1}{2} A_{H}(V, V)-(f, V) .
$$

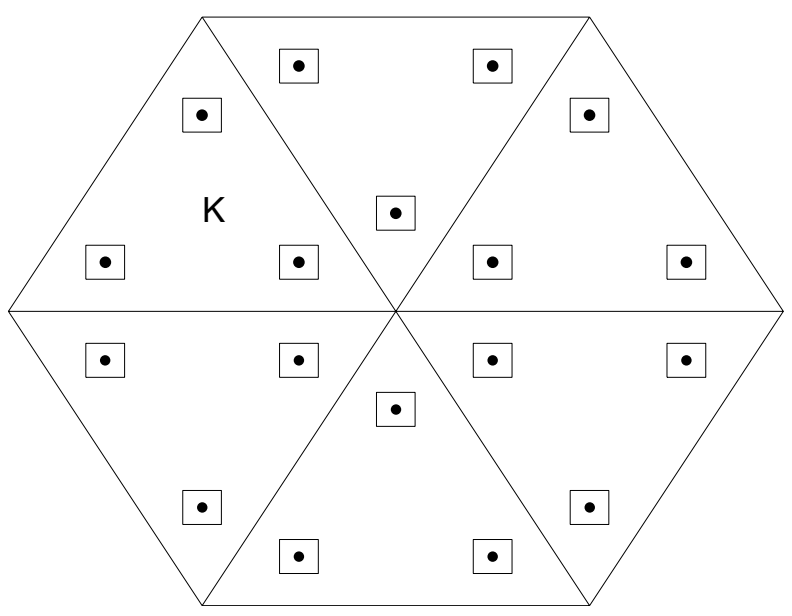

Figure 1. Illustration of HMM for solving (1.1). The dots are the quadrature points. The little squares are the microcell $I_{\delta}\left(x_{\ell}\right)$.

To summarize, HMM has the following features:

(1) It gives a framework that allows us to maximally take advantage of the special features of the problem such as scale separation. For periodic homogenization problems, the cost of HMM is comparable to the special techniques discussed in $[3,7,29,35$. However HMM is also applicable for random problems and for problems whose coefficient $a^{\varepsilon}(x)$ does not has the structure of $a(x, x / \varepsilon)$. For problems without scale separation, we may consider other possible special features of the problem such as local self-similarity, which is considered in [19].

(2) For problems without any special features, HMM becomes a fine scale solver by choosing an $H$ that resolves the fine scales and letting $\mathcal{A}_{H}(x)=a^{\varepsilon}(x)$.

Some related ideas exist in the literature. Durlofsky 14 proposed an up-scaling method, which directly solves some local problems for obtaining the effective coefficients [33, 40, 41. Oden and Vemaganti [35] proposed a method that aims at recovering the oscillations in $\nabla u^{\varepsilon}$ locally by solving a local problem with some given approximation to the macroscopic state $U$ as the boundary condition. This idea is sometimes used in HMM to recover the microstructural information. Other numerical methods that use local microscale solvers to help extract macroscale behavior are found in [26] 27].

The numerical performance of HMM including comparison with other methods is discussed in [31].

This paper will focus on the analysis of HMM. We will estimate the error between the numerical solutions of HMM and the solutions of (1.4). We will also discuss 
how to construct better approximations of $u^{\varepsilon}$ from the HMM solutions. Our basic strategy is as follows. First we will prove a general statement that the error between the HMM solution and the solution of (1.4) is controlled by the standard error in the macroscale solver plus a new term, called $e(\mathrm{HMM})$, due to the error in estimating the stiffness matrix. We then estimate $e(\mathrm{HMM})$. This second part is only done for either periodic or random homogenization problems, since concrete results are only possible if the behavior of $u^{\varepsilon}$ is well understood. We believe that this overall strategy will be useful for analyzing other multiscale methods.

We will always assume that $a^{\varepsilon}(x)$ is smooth, symmetric and uniformly elliptic:

$$
\lambda I \leq a^{\varepsilon} \leq \Lambda I
$$

for some $\lambda, \Lambda>0$. We will use the summation convention and standard notation for Sobolev spaces (see [1]). We will use $|\cdot|$ to denote the absolute value of a scalar quantity, the Euclidean norm of a vector and the volume of a set $K$. For the quadrature formula (1.5), we will assume the following accuracy conditions for $k$ th-order numerical quadrature scheme [1]:

$$
f_{K} p(x) d x:=\frac{1}{|K|} \int_{K} p(x) d x=\sum_{\ell=1}^{L} \omega_{\ell} p\left(x_{\ell}\right) \quad \text { for all } p(x) \in \mathcal{P}_{2 k-2} .
$$

Here $\omega_{\ell}>0$, for $\ell=1, \cdots, L$. For $k=1$, we assume the above formula to be exact for $p \in \mathcal{P}_{1}$.

1.3. Main results. Our main results for the linear problem are as follows.

Theorem 1.1. Denote by $U_{0}$ and $U_{\mathrm{HMM}}$ the solution of (1.4) and the HMM solution, respectively. Let

$$
e(\mathrm{HMM})=\max _{\substack{x_{\ell} \in K \\ K \in \mathcal{T}_{H}}}\left\|\mathcal{A}\left(x_{\ell}\right)-\mathcal{A}_{H}\left(x_{\ell}\right)\right\|,
$$

where $\|\cdot\|$ is the Euclidean norm. If $U_{0}$ is sufficiently smooth and (1.10) holds, then there exists a constant $C$ independent of $\varepsilon, \delta$ and $H$, such that

$$
\begin{aligned}
& \left\|U_{0}-U_{\mathrm{HMM}}\right\|_{1} \leq C\left(H^{k}+e(\mathrm{HMM})\right), \\
& \left\|U_{0}-U_{\mathrm{HMM}}\right\|_{0} \leq C\left(H^{k+1}+e(\mathrm{HMM})\right) .
\end{aligned}
$$

If there exits a constant $C_{0}$ such that $e(\mathrm{HMM})|\ln H|<C_{0}$, then there exists a constant $H_{0}$ such that for all $H \leq H_{0}$,

$$
\left\|U_{0}-U_{\text {HMM }}\right\|_{1, \infty} \leq C\left(H^{k}+e(\mathrm{HMM})\right)|\ln H| .
$$

At this stage, no assumption on the form of $a^{\varepsilon}(x)$ is necessary. $U_{0}$ can be the solution of an arbitrary macroscopic equation with the same right-hand side as in (1.1). Of course for $U_{\mathrm{HMM}}$ to converge to $U_{0}$, i.e., $e(\mathrm{HMM}) \rightarrow 0, U_{0}$ must be chosen as the solution of the homogenized equation, which we now assume exists. To obtain quantitative estimates on $e(\mathrm{HMM})$, we must restrict ourselves to more specific cases.

Theorem 1.2. For the periodic homogenization problem, we have

$$
e(\mathrm{HMM}) \leq \begin{cases}C \varepsilon & \text { if } I_{\delta}\left(x_{\ell}\right)=x_{\ell}+\varepsilon I, \\ C\left(\frac{\varepsilon}{\delta}+\delta\right) & \text { otherwise. }\end{cases}
$$


In the first case, we replace the boundary condition in (1.6) by a periodic boundary condition: $v_{\ell}^{\varepsilon}-V_{\ell}$ is periodic with period $\varepsilon I$. For the second result we do not need to assume that the period of $a(x, \cdot)$ is a cube: In fact it can be of arbitrary shape as long as its translation tiles up the whole space.

Another important case for which a specific estimate on $e(\mathrm{HMM})$ can be obtained is the random homogenization. In this case, using results in [43, we have

Theorem 1.3. For the random homogenization problem, assuming that $(\mathbf{A})$ in the Appendix holds (see [43]), we have

$$
\mathbb{E} e(\mathrm{HMM}) \leq \begin{cases}C(\kappa)\left(\frac{\varepsilon}{\delta}\right)^{\kappa} & d=3, \\ \text { remains open } & d=2, \\ C(\kappa)\left(\frac{\varepsilon}{\delta}\right)^{1 / 2} & d=1,\end{cases}
$$

where

$$
\kappa=\frac{6-12 \gamma}{25-8 \gamma}
$$

for any $0<\gamma<1 / 2$. By choosing $\gamma$ small, $\kappa$ can be arbitrarily close to $6 / 25$.

The probabilistic set-up will be given in the Appendix. To prove this result, we assume that 1.8a) is used with $\delta^{\prime}=\delta / 2$.

1.4. Recovering the microstructural information. In many applications, the microstructure information in $u^{\varepsilon}(x)$ is very important. $U_{\text {HMM }}$ by itself does not give this information. However, this information can be recovered using a simple post-processing technique. For the general case, having $U_{\mathrm{HMM}}$, one can obtain locally the microstructural information using an idea in [35]. Assume that we are interested in recovering $u^{\varepsilon}$ and $\nabla u^{\varepsilon}$ only in the subdomain $\Omega \subset D$. Consider the following auxiliary problem:

$$
\left\{\begin{aligned}
-\operatorname{div}\left(a^{\varepsilon}(x) \nabla \tilde{u}^{\varepsilon}(x)\right) & =f(x) & & x \in \Omega_{\eta}, \\
\tilde{u}^{\varepsilon}(x) & =U_{\mathrm{HMM}}(x) & & x \in \partial \Omega_{\eta},
\end{aligned}\right.
$$

where $\Omega_{\eta}$ satisfies $\Omega \subset \Omega_{\eta} \subset D$ and $\operatorname{dist}\left(\partial \Omega, \partial \Omega_{\eta}\right)=\eta$. We then have

Theorem 1.4. There exists a constant $C$ such that

$$
\left(f_{\Omega}\left|\nabla\left(u^{\varepsilon}-\tilde{u}^{\varepsilon}\right)\right|^{2} d x\right)^{1 / 2} \leq \frac{C}{\eta}\left(\left\|U_{0}-U_{\mathrm{HMM}}\right\|_{L^{\infty}\left(\Omega_{\eta}\right)}+\left\|u^{\varepsilon}-U_{0}\right\|_{L^{\infty}\left(\Omega_{\eta}\right)}\right) .
$$

For the random problem, the last term was estimated in [43].

A much simpler procedure exists for the periodic homogenization problem. Consider the case when $k=1$ and choose $I_{\delta}=x_{K}+\varepsilon I$, where $x_{K}$ is the barycenter of $K$. Here we have assumed that the quadrature point is at $x_{K}$.

Let $\tilde{u}^{\varepsilon}$ be defined piecewise as follows:

(1) $\left.\tilde{u}^{\varepsilon}\right|_{I_{\delta}}=v_{K}^{\varepsilon}$, where $v_{K}^{\varepsilon}$ is the solution of (1.6) with the boundary condition that $v_{K}^{\varepsilon}-U_{\mathrm{HMM}}$ is periodic with period $\varepsilon I$ and $\int_{I_{\delta}}\left(\tilde{u}^{\varepsilon}-U_{\mathrm{HMM}}\right)(x) d x=0$.

(2) $\left.\left(\tilde{u}^{\varepsilon}-U_{\mathrm{HMM}}\right)\right|_{K}$ is periodic with period $\varepsilon I$. 
For this case, we can prove

Theorem 1.5. Let $\tilde{u}^{\varepsilon}$ be defined as above. Then

$$
\left(\sum_{K \in \mathcal{T}_{H}}\left\|\nabla\left(u^{\varepsilon}-\tilde{u}^{\varepsilon}\right)\right\|_{0, K}^{2}\right)^{1 / 2} \leq C(\sqrt{\varepsilon}+H) .
$$

Similar results with some modification hold for nonlinear problems. The details are given in $\S 5$.

1.5. Some technical background. In this subsection, we will list some general results that will be frequently referred to later on.

Given a triangulation $\mathcal{T}_{H}$, it is called regular if there is a constant $\sigma$ such that

$$
\frac{H_{K}}{\rho_{K}} \leq \sigma \quad \text { for all } K \in \mathcal{T}_{H}
$$

and if the quantity

$$
H=\max _{K \in \mathcal{T}_{H}} H_{K}
$$

approaches zero, where $H_{K}$ is the diameter of $K$ and $\rho_{K}$ is the diameter of the largest ball inscribed in $K . \mathcal{T}_{H}$ satisfies an inverse assumption if there exists a constant $\nu$ such that

$$
\frac{H}{H_{K}} \leq \nu \quad \text { for all } K \in \mathcal{T}_{H}
$$

A regular family of triangulation of $\mathcal{T}_{H}$ satisfying the inverse assumption is called quasi-uniform.

The following interpolation result for the Lagrange finite element is adapted from 10. Here and in what follows, for any $k \geq 2, \nabla^{k} v$ is understood in a piecewise manner.

Theorem 1.6 ([10]). Let $\Pi$ be $k$ th-order Lagrange interpolate operator, and assume that the following inclusions hold:

$$
W^{k+1, p}(\hat{K}) \hookrightarrow \mathcal{C}^{0}(\hat{K}) \quad \text { and } \quad W^{k+1, p}(\hat{K}) \hookrightarrow W^{m, q}(\hat{K}) .
$$

Then

$$
|v-\Pi v|_{m, q, K} \leq C|K|^{1 / q-1 / p} \frac{H_{K}^{k+1}}{\rho_{K}^{m}}|v|_{k+1, p, K} .
$$

If $\mathcal{T}_{H}$ is regular, we have the global estimate

$$
|v-\Pi v|_{m, q, D} \leq C H^{k+1-m+\min \{0, d(1 / q-1 / p)\}}|v|_{k+1, p, D} .
$$

Inequality (1.18) is proven in 10, Theorem 3.1.6], and (1.19) is a direct consequence of (1.18) and the inverse inequality below.

Using (1.19) with $p=q=2$ and $m=2, k=1$, we have $\|v-\Pi v\|_{2, D} \leq C\|v\|_{2, D}$. Hence

$$
\|\Pi v\|_{2, D} \leq\|v-\Pi v\|_{2, D}+\|v\|_{2, D} \leq C\|v\|_{2, D} .
$$

We will also need the following form of the inverse inequality.

Theorem 1.7 ([10, Theorem 3.2.6]). Assume that $\mathcal{T}_{H}$ is regular, and assume also that the two pairs $(l, r)$ and $(m, q)$ with $l, m \geq 0$ and $r, q \in[0, \infty]$ satisfy

$$
l \leq m \quad \text { and } \quad \mathcal{P}_{k}(\hat{K}) \subset W^{l, r}(\hat{K}) \cap W^{m, q}(\hat{K})
$$


Then there exists a constant $C=C(\sigma, \nu, l, r, m, q)$ such that

$$
|v|_{m, q, K} \leq C H_{K}^{l-m+d(1 / q-1 / r)}|v|_{l, r, K}
$$

for any $v \in \mathcal{P}_{k}(\hat{K})$.

If in addition $\mathcal{T}_{H}$ satisfies the inverse assumption, then there exists a constant $C=C(\sigma, \nu, l, r, m, q)$ such that

$$
\left(\sum_{K \in \mathcal{T}_{H}}|v|_{m, q, K}^{q}\right)^{1 / q} \leq C H^{l-m+\min \{0, d(1 / q-1 / r)\}}\left(\sum_{K \in \mathcal{T}_{H}}|v|_{l, r, K}^{r}\right)^{1 / r}
$$

for any $v \in X_{H}$ and $r, q<\infty$, with

$$
\begin{array}{ccc}
\max _{K \in \mathcal{T}_{H}}|v|_{m, \infty, K} \quad \text { replacing } & \left(\sum_{K \in \mathcal{T}_{H}}|v|_{m, q, K}^{q}\right)^{1 / q}, \quad \text { if } q=\infty, \\
\max _{K \in \mathcal{T}_{H}}|v|_{l, \infty, K} \quad \text { replacing } & \left(\sum_{K \in \mathcal{T}_{H}}|v|_{l, r, K}^{r}\right)^{1 / r}, \quad \text { if } r=\infty .
\end{array}
$$

The following simple result will be used repeatedly.

Lemma 1.8. Let $A_{1}(x)$ and $A_{2}(x)$ be symmetric matrices satisfying (1.9). Let $\varphi_{1}$ be the solution of

$$
-\operatorname{div}\left(A_{1}(x) \nabla \varphi_{1}(x)\right)=\operatorname{div}\left(\tilde{A}_{1}(x) \nabla F_{1}(x)\right) \quad x \in \Omega,
$$

with either the Dirichlet or periodic boundary condition on $\partial \Omega$. Let $\varphi_{2}$ be a solution of (1.23) with $A_{1}, \tilde{A}_{1}$ and $F_{1}$ replaced by $A_{2}, \tilde{A}_{2}$ and $F_{2}$, respectively, and let $\varphi_{2}$ satisfy the same boundary condition as $\varphi_{1}$. Then

$\lambda\left\|\nabla\left(\varphi_{1}-\varphi_{2}\right)\right\|_{0, \Omega} \leq \max _{x \in \Omega}\left|\left(\tilde{A}_{1}-\tilde{A}_{2}\right)(x)\right|\left\|\nabla F_{1}\right\|_{0, \Omega}+\max _{x \in \Omega}\left|\left(A_{1}-A_{2}\right)(x)\right|\left\|\nabla \varphi_{2}\right\|_{0, \Omega}$

$$
+\max _{x \in \Omega}\left|\tilde{A}_{2}(x)\right|\left\|\nabla\left(F_{1}-F_{2}\right)\right\|_{0, \Omega} .
$$

Proof. Inequality (1.24) is a direct consequence of

$\lambda\left\|\nabla\left(\varphi_{1}-\varphi_{2}\right)\right\|_{0, \Omega}^{2} \leq \int_{\Omega} \nabla\left(\varphi_{1}-\varphi_{2}\right) \cdot\left(\left(\tilde{A}_{2}-\tilde{A}_{1}\right) \nabla F_{1}+\left(A_{2}-A_{1}\right) \nabla \varphi_{2}+\tilde{A}_{2} \nabla\left(F_{2}-F_{1}\right)\right)$.

The following simple result underlies the stability of HMM for problem (1.1).

Lemma 1.9. Let $\varphi$ be the solution of

$$
\left\{\begin{aligned}
-\operatorname{div}(a \nabla \varphi) & =0 & & \text { in } \Omega \subset \mathbb{R}^{d}, \\
\varphi & =V_{\ell} & & \text { on } \in \partial \Omega,
\end{aligned}\right.
$$

where $V_{\ell}$ is a linear function and $a=\left(a_{i j}\right)$ satisfies

$$
\lambda I \leq a \leq \Lambda I .
$$

Then we have

$$
\left\|\nabla V_{\ell}\right\|_{0, \Omega} \leq\|\nabla \varphi\|_{0, \Omega} \quad \text { and } \quad\left(\int_{\Omega} \nabla \varphi \cdot a \nabla \varphi\right)^{1 / 2} \leq\left(\int_{\Omega} \nabla V_{\ell} \cdot a \nabla V_{\ell}\right)^{1 / 2} .
$$


Proof. Notice that $\varphi=V_{\ell}$ on the edges of $\Omega$, using the fact that $\nabla V_{\ell}$ is a constant in $\Omega$, and integration by parts leads to

$$
\int_{\Omega} \nabla\left(\varphi-V_{\ell}\right)(x) \nabla V_{\ell}(x) d x=0,
$$

which implies

$$
\int_{\Omega}|\nabla \varphi(x)|^{2} d x=\int_{\Omega}\left|\nabla V_{\ell}(x)\right|^{2} d x+\int_{\Omega}\left|\nabla\left(\varphi-V_{\ell}\right)(x)\right|^{2} d x .
$$

This gives the first result in (1.26). Multiplying (1.25) by $\varphi(x)-V_{\ell}(x)$ and integrating by parts, we obtain

$$
\begin{aligned}
\int_{\Omega} \nabla \varphi(x) \cdot a \nabla \varphi(x) d x & +\int_{\Omega} \nabla\left(\varphi-V_{\ell}\right)(x) \cdot a \nabla\left(\varphi-V_{\ell}\right)(x) d x \\
& =\int_{\Omega} \nabla V_{\ell}(x) \cdot a \nabla V_{\ell}(x) d x .
\end{aligned}
$$

This gives the second part of (1.26).

Remark 1.10. For this result, the coefficient $a=\left(a_{i j}\right)$ may depend on the solution, i.e., (1.25) may be nonlinear.

Remark 1.11. The same result holds if we use instead a periodic boundary condition: $\varphi-V_{\ell}$ is periodic with period $\Omega$.

\section{Generalities}

Here we prove Theorem 1.1. We will let $U_{H}=U_{\text {HMM }}$ for convenience.

Since $U_{H}$ is the numerical solution associated with the quadratic form $A_{H}, U_{0}$ is the exact solution associated with the quadratic form $A$, defined for any $V \in H_{0}^{1}(D)$ as

$$
A(V, V)=\int_{D} \nabla V(x) \cdot \mathcal{A}(x) \nabla V(x) d x .
$$

To estimate $U_{0}-U_{H}$, we view $A_{H}$ as an approximation to $A$, and we use Strang's first lemma [10].

Using (1.26) with $\Omega=I_{\delta}\left(x_{\ell}\right)$ and (1.9), for any $V \in X_{H}$, we have

$$
\begin{aligned}
A_{H}(V, V) & \geq \lambda \sum_{K \in \mathcal{T}_{H}}|K| \sum_{x_{\ell} \in K} \omega_{\ell} f_{I_{\delta}\left(x_{\ell}\right)}\left|\nabla V_{\ell}(x)\right|^{2} d x \\
& =\lambda \sum_{K \in \mathcal{T}_{H}}|K| \sum_{x_{\ell} \in K} \omega_{\ell}\left|\nabla V\left(x_{\ell}\right)\right|^{2} \\
& =\lambda\|\nabla V\|_{0}^{2} .
\end{aligned}
$$


Similarly, for any $V, W \in X_{H}$, we obtain

$$
\begin{aligned}
\left|A_{H}(V, W)\right| & \leq \sum_{K \in \mathcal{T}_{H}}|K| \sum_{x_{\ell} \in K} \omega_{\ell}\left(f_{I_{\delta}\left(x_{\ell}\right)} \nabla V_{\ell} \cdot a^{\varepsilon} \nabla V_{\ell}\right)^{\frac{1}{2}}\left(f_{I_{\delta}\left(x_{\ell}\right)} \nabla W_{\ell} \cdot a^{\varepsilon} \nabla W_{\ell}\right)^{\frac{1}{2}} \\
& \leq \Lambda \sum_{K \in \mathcal{T}_{H}}|K| \sum_{x_{\ell} \in K} \omega_{\ell}\left|\nabla V\left(x_{\ell}\right)\right|\left|\nabla W\left(x_{\ell}\right)\right| \\
& =\Lambda \sum_{K \in \mathcal{T}_{H}} \int_{K}|\nabla V(x)||\nabla W(x)| d x \\
& \leq \Lambda\|\nabla V\|_{0}\|\nabla W\|_{0} .
\end{aligned}
$$

The existence and the uniqueness of the solutions to (1.8) follow from (2.1) and (2.2) via the Lax-Milgram lemma and the Poincaré inequality.

To streamline the proof of Theorem 1.1, we introduce the following auxiliary bilinear form $\hat{A}_{H}$.

$$
\hat{A}_{H}(V, W)=\sum_{K \in \mathcal{T}_{H}} \hat{A}_{K}(V, W) \quad \text { with } \quad \hat{A}_{K}(V, W)=|K| \sum_{x_{\ell} \in K} \omega_{\ell}(\nabla W \cdot \mathcal{A} \nabla V)\left(x_{\ell}\right) .
$$

Classical results on numerical integration [11, Theorem 6] give for any $V, W \in$ $X_{H}$,

$$
\left|\hat{A}_{K}(V, W)-\int_{K} \nabla W \cdot \mathcal{A} \nabla V d x\right| \leq C H^{m}\|V\|_{m, K}\|\nabla W\|_{0, K} \quad 1 \leq m \leq k .
$$

Moreover, for any $V, W \in X_{H}$, if $\|V\|_{k+1}$ and $\|W\|_{2}$ are bounded, we have [11] Theorem 8],

$$
\left|\hat{A}_{H}(V, W)-A(V, W)\right| \leq C H^{k+1}\|V\|_{k+1}\|W\|_{2} .
$$

Proof of Theorem 1.1. Using the first Strang lemma [10, Theorem 4.1.1], we have

$$
\left\|U_{0}-U_{H}\right\|_{1} \leq C \inf _{V \in X_{H}}\left(\left\|U_{0}-V\right\|_{1}+\sup _{W \in X_{H}} \frac{\left|A_{H}(V, W)-A(V, W)\right|}{\|W\|_{1}}\right) .
$$

Let $V=\Pi U_{0}$ and using (1.19) with $m=1, p=q=2$, we have

$$
\inf _{V \in X_{H}}\left\|U_{0}-V\right\|_{1} \leq\left\|U_{0}-\Pi U_{0}\right\|_{1} \leq C H^{k} .
$$

It remains to estimate $\left|A_{H}(V, W)-A(V, W)\right|$ for $V=\Pi U_{0}$ and $W \in X_{H}$. Using (2.3), we get

$$
\begin{aligned}
\left|A_{H}(V, W)-A(V, W)\right| & \leq\left|A_{H}(V, W)-\hat{A}_{H}(V, W)\right|+\left|\hat{A}_{H}(V, W)-A(V, W)\right| \\
& \leq\left(e(\mathrm{HMM})\|\nabla V\|_{0}+C H^{k}\|V\|_{k}\right)\|\nabla W\|_{0} .
\end{aligned}
$$

This gives (1.11)

To get the $\mathrm{L}^{2}$ estimate, we use the Aubin-Nitsche dual argument [10. To this end, consider the following auxiliary problem: Find $w \in H_{0}^{1}(D)$ such that

$$
A(v, w)=\left(U_{0}-U_{H}, v\right) \quad \text { for all } v \in H_{0}^{1}(D) .
$$

The standard regularity result reads 24$]$

$$
\|w\|_{2} \leq C\left\|U_{0}-U_{H}\right\|_{0} .
$$


Putting $v=U_{0}-U_{H}$ into the right-hand side of (2.7), we obtain

$$
\begin{aligned}
\left\|U_{0}-U_{H}\right\|_{0}^{2} & =A\left(U_{0}-U_{H}, w-\Pi w\right)+\left(A_{H}\left(U_{H}, \Pi w\right)-A\left(U_{H}, \Pi w\right)\right) \\
& =A\left(U_{0}-U_{H}, w-\Pi w\right) \\
& +\left(A_{H}\left(U_{H}-\Pi U_{0}, \Pi w\right)-A\left(U_{H}-\Pi U_{0}, \Pi w\right)\right) \\
& +\left(A_{H}\left(\Pi U_{0}, \Pi w\right)-A\left(\Pi U_{0}, \Pi w\right)\right) .
\end{aligned}
$$

Using (2.6) with $k=1$, we bound the first two terms in the right-hand side of the above identity as

$$
\left|A\left(U_{0}-U_{H}, w-\Pi w\right)\right| \leq C\left\|U_{0}-U_{H}\right\|_{1}\|w-\Pi w\|_{1} \leq C H\left\|U_{0}-U_{H}\right\|_{1}\|w\|_{2}
$$

and

$$
\left|A_{H}\left(U_{H}-\Pi U_{0}, \Pi w\right)-A\left(U_{H}-\Pi U_{0}, \Pi w\right)\right| \leq(e(\mathrm{HMM})+C H)\left\|U_{0}-U_{H}\right\|_{1}\|\Pi w\|_{1} .
$$

The last term in the right-hand side of (2.9) may be decomposed into

$$
\begin{aligned}
A_{H}\left(\Pi U_{0}, \Pi w\right)-A\left(\Pi U_{0}, \Pi w\right) & =\left(A_{H}\left(\Pi U_{0}, \Pi w\right)-\hat{A}_{H}\left(\Pi U_{0}, \Pi w\right)\right) \\
& +\left(\hat{A}_{H}\left(\Pi U_{0}, \Pi w\right)-A\left(\Pi U_{0}, \Pi w\right)\right) .
\end{aligned}
$$

It follows from (2.4) that

$$
\left|\hat{A}_{H}\left(\Pi U_{0}, \Pi w\right)-A\left(\Pi U_{0}, \Pi w\right)\right| \leq C H^{k+1}\left\|U_{0}\right\|_{k+1}\|w\|_{2} .
$$

By definition of $e(\mathrm{HMM})$ and using (1.20), we get

$$
\left|A_{H}\left(\Pi U_{0}, \Pi w\right)-\hat{A}_{H}\left(\Pi U_{0}, \Pi w\right)\right| \leq C e(\mathrm{HMM})\left\|\nabla \Pi U_{0}\right\|_{0}\|w\|_{2} .
$$

Combining the above estimates and using (2.8) lead to (1.12).

It remains to prove (1.13). As in 37, for any point $z \in D$, we define the regularized Green's function $G^{z} \in H_{0}^{1}(D)$ and the discrete Green's function $G_{H}^{z} \in$ $X_{H}$ as

$$
\begin{aligned}
A\left(G^{z}, V\right)=\left(\delta_{z}, \partial V\right) & \text { for all } V \in H_{0}^{1}(D), \\
A\left(G_{H}^{z}, V\right)=\left(\delta_{z}, \partial V\right) & \text { for all } V \in X_{H},
\end{aligned}
$$

where $\delta_{z}$ is the regularized Dirac- $\delta$ function defined in [37. It is well known that

$$
\left\|G^{z}-G_{H}^{z}\right\|_{1,1} \leq C \quad \text { and } \quad\left\|G_{H}^{z}\right\|_{1,1} \leq C|\ln H| .
$$

A proof for (2.11) can be obtained by using the weighted-norm technique [37. We refer to [9, Chapter 7] for details. Using the definition of $G^{z}$ and $G_{H}^{z}$, a simple manipulation gives

$$
\begin{aligned}
\partial\left(U_{0}-U_{H}\right)(z)= & A\left(G^{z}, U_{0}-\Pi U_{0}\right)+A\left(G^{z}, \Pi U_{0}-U_{H}\right) \\
= & A\left(G^{z}-G_{H}^{z}, U_{0}-\Pi U_{0}\right)+A\left(G_{H}^{z}, U_{0}-U_{H}\right) \\
= & A\left(G^{z}-G_{H}^{z}, U_{0}-\Pi U_{0}\right)+A_{H}\left(U_{H}, G_{H}^{z}\right)-A\left(U_{H}, G_{H}^{z}\right) \\
= & A\left(G^{z}-G_{H}^{z}, U_{0}-\Pi U_{0}\right)+\left(A_{H}\left(\Pi U_{0}, G_{H}^{z}\right)-A\left(\Pi U_{0}, G_{H}^{z}\right)\right) \\
& +\left(A_{H}\left(U_{H}-\Pi U_{0}, G_{H}^{z}\right)-A\left(U_{H}-\Pi U_{0}, G_{H}^{z}\right)\right) .
\end{aligned}
$$

Using (2.11), we obtain

$$
\begin{aligned}
\left\|U_{0}-U_{H}\right\|_{1, \infty} \leq & C\left\|U_{0}-\Pi U_{0}\right\|_{1, \infty}+\left|A\left(\Pi U_{0}, G_{H}^{z}\right)-A_{H}\left(\Pi U_{0}, G_{H}^{z}\right)\right| \\
& +\left|A\left(U_{H}-\Pi U_{0}, G_{H}^{z}\right)-A_{H}\left(U_{H}-\Pi U_{0}, G_{H}^{z}\right)\right| .
\end{aligned}
$$


Using (2.60), we get

$$
\begin{gathered}
\left|A\left(\Pi U_{0}, G_{H}^{z}\right)-A_{H}\left(\Pi U_{0}, G_{H}^{z}\right)\right| \leq\left(e(\mathrm{HMM})+C H^{k}\right) \sum_{K \in \mathcal{T}_{H}}\left\|\Pi U_{0}\right\|_{k, K}\left\|\nabla G_{H}^{z}\right\|_{0, K} \\
\leq C\left(e(\mathrm{HMM})+H^{k}\right) \sum_{K \in \mathcal{T}_{H}}\left\|\Pi U_{0}\right\|_{k, \infty, K}\left\|\nabla G_{H}^{z}\right\|_{L^{1}(K)} \\
\leq C\left(e(\mathrm{HMM})+H^{k}\right)|\ln H|\left\|U_{0}\right\|_{k+1, \infty},
\end{gathered}
$$

where we have used the inverse inequality (1.21).

Similarly, we have

$$
\begin{aligned}
& \left|A\left(U_{H}-\Pi U_{0}, G_{H}^{z}\right)-A_{H}\left(U_{H}-\Pi U_{0}, G_{H}^{z}\right)\right| \\
\leq & (e(\mathrm{HMM})+C H) \sum_{K \in \mathcal{T}_{H}}\left\|U_{H}-\Pi U_{0}\right\|_{1, K}\left\|\nabla G_{H}^{z}\right\|_{0, K} \\
\leq & C(e(\mathrm{HMM})+H) \sum_{K \in \mathcal{T}_{H}}\left\|U_{H}-\Pi U_{0}\right\|_{1, \infty, K}\left\|\nabla G_{H}^{z}\right\|_{0,1, K} \\
\leq & C(e(\mathrm{HMM})+H)|\ln H|\left\|U_{0}-U_{H}\right\|_{1, \infty} \\
& +C(e(\mathrm{HMM})+H)|\ln H| H^{k}\left\|U_{0}\right\|_{k+1, \infty} .
\end{aligned}
$$

A combination of the above three estimates yields

$$
\begin{aligned}
\left\|U_{0}-U_{H}\right\|_{1, \infty} \leq & C H^{k}+C(e(\mathrm{HMM})+H)|\ln H|\left\|U_{0}-U_{H}\right\|_{1, \infty} \\
& +C\left(e(\mathrm{HMM})+H^{k}\right)|\ln H|\left\|U_{0}\right\|_{k+1, \infty} .
\end{aligned}
$$

If $e(\mathrm{HMM})|\ln H|<C_{0}:=1 /(2 C)$, then there exits a constant $H_{0}$ such that for all $H \leq H_{0}$,

$$
C(e(\mathrm{HMM})+H)|\ln H| \leq 1 / 2+C H|\ln H|<1 .
$$

We thus obtain (1.13) and this completes the proof.

Combining the foregoing proof for the $\mathrm{L}^{2}$ and $\mathrm{W}^{1, \infty}$ estimates, using the Green's function defined in 39], we obtain

Remark 2.1. Under the same condition for the $\mathrm{W}^{1, \infty}$ estimate in Theorem 1.1, we have

$$
\left\|U_{0}-U_{H}\right\|_{L^{\infty}} \leq C\left(e(\mathrm{HMM})+H^{k+1}\right)|\ln H|^{2} .
$$

\section{Estimating e(HMM)}

In this section, we estimate $e(\mathrm{HMM})$ for problems with locally periodic coefficients. The estimate of $e(\mathrm{HMM})$ for problems with random coefficients can be found in the Appendix.

We assume that $a^{\varepsilon}(x)=a(x, x / \varepsilon)$, where $a^{\varepsilon}$ is smooth in $x$ and periodic in $y$ with period $I$. Define $\kappa=\lfloor\delta / \varepsilon\rfloor$, and we introduce $\hat{V}_{\ell}$ as

$$
\hat{V}_{\ell}(x)=V_{\ell}(x)+\varepsilon \chi^{k}\left(x_{\ell}, \frac{x}{\varepsilon}\right) \frac{\partial V_{\ell}}{\partial x_{k}}(x),
$$

where $\left\{\chi^{j}\right\}_{j=1}^{d}$ is defined as: For $j=1, \cdots, d, \chi^{j}(x, y)$ is periodic in $y$ with period $I$ and satisfies

$$
-\frac{\partial}{\partial y_{i}}\left(a_{i k} \frac{\partial \chi^{j}}{\partial y_{k}}\right)(x, y)=\frac{\partial}{\partial y_{i}} a_{i j}(x, y) \quad \text { in } I, \quad \int_{I} \chi^{j}(x, y) d y=0 .
$$


Given $\left\{\chi^{j}\right\}_{j=1}^{d}$, the homogenized coefficient $\mathcal{A}=\left(\mathcal{A}_{i j}(x)\right)$ is given by

$$
\mathcal{A}_{i j}(x)=f_{I}\left(a_{i j}+a_{i k} \frac{\partial \chi^{j}}{\partial y_{k}}\right)(x, y) d y .
$$

Note that $\left\{\chi^{j}\right\}_{j=1}^{d}$ is smooth and bounded in all norms.

First let us consider the case when $I_{\delta}\left(x_{\ell}\right)=x_{\ell}+\varepsilon I$, and (1.6) is solved with the periodic boundary condition. Denote by $\hat{v}_{\ell}^{\varepsilon}$ the solution of (1.6) with the coefficients $a^{\varepsilon}(x)$ replaced by $a\left(x_{\ell}, x / \varepsilon\right)$. $\hat{v}_{\ell}^{\varepsilon}$ may be viewed as a perturbation of $v_{\ell}^{\varepsilon}$. Using Lemma 1.8 we get

$$
\left\|\nabla\left(v_{\ell}^{\varepsilon}-\hat{v}_{\ell}^{\varepsilon}\right)\right\|_{0, I_{\varepsilon}} \leq C \varepsilon\left\|\nabla V_{\ell}\right\|_{0, I_{\varepsilon}}
$$

Observe that $\hat{v}_{\ell}^{\varepsilon}=\hat{V}_{\ell}$. A direct calculation yields

$$
\begin{aligned}
\left(\nabla W \cdot\left(\mathcal{A}_{H}-\mathcal{A}\right) \nabla V\right)\left(x_{\ell}\right)= & \int_{I_{\varepsilon}} \nabla w_{\ell}^{\varepsilon} \cdot\left[a\left(x, \frac{x}{\varepsilon}\right)-a\left(x_{\ell}, \frac{x}{\varepsilon}\right)\right] \nabla v_{\ell}^{\varepsilon} d x \\
& +f_{I_{\varepsilon}} \nabla w_{\ell}^{\varepsilon} \cdot a\left(x_{\ell}, \frac{x}{\varepsilon}\right) \nabla\left(v_{\ell}^{\varepsilon}-\hat{v}_{\ell}^{\varepsilon}\right) d x .
\end{aligned}
$$

Using (3.3), we get

$$
e(\mathrm{HMM}) \leq C \varepsilon
$$

Next we consider the more general case when $I_{\delta}$ is a cube of size $\delta$ not necessarily equal to $\varepsilon$. The following analysis applies equally well to the case when the period of $a(x, \cdot)$ is of general and even nonpolygonal shape. This situation arises in some examples of composite materials [30]. We will show that if $\delta$ is much larger than $\varepsilon$, then the averaged energy density for the solution of (1.6) closely approximates the energy density of the homogenized problem. We begin with the following observation:

$$
\begin{aligned}
(\nabla W \cdot \mathcal{A} \nabla V)\left(x_{\ell}\right) & =\nabla W_{\ell}(x) \cdot \mathcal{A}\left(x_{\ell}\right) \nabla V_{\ell}(x) \\
& =f_{I_{\kappa \varepsilon}\left(x_{\ell}\right)} \nabla W_{\ell} \cdot a\left(x_{\ell}, \frac{x}{\varepsilon}\right) \nabla \hat{V}_{\ell} d x .
\end{aligned}
$$

We first establish some estimates on the solution of the cell problem (1.6). We will write $I_{\delta}$ instead of $I_{\delta}\left(x_{\ell}\right)$ if there is no risk of confusion.

Lemma 3.1. There exists a constant $C$ independent of $\varepsilon$ and $\delta$ such that for each $\ell$

$$
\left\|\nabla v_{\ell}^{\varepsilon}\right\|_{0, I_{\delta} \backslash I_{\kappa \varepsilon}} \leq C\left(\left(\frac{\varepsilon}{\delta}\right)^{1 / 2}+\delta\right)\left\|\nabla V_{\ell}\right\|_{0, I_{\delta}} .
$$

Proof. We still denote by $\hat{v}_{\ell}^{\varepsilon}$ the solution of (1.6) with the coefficient $a^{\varepsilon}(x)$ replaced by $a\left(x_{\ell}, x / \varepsilon\right)$. Using Lemma 1.8, we get

$$
\left\|\nabla\left(v_{\ell}^{\varepsilon}-\hat{v}_{\ell}^{\varepsilon}\right)\right\|_{0, I_{\delta}} \leq C \delta\left\|\nabla V_{\ell}\right\|_{0, I_{\delta}} .
$$

Define $\theta_{\ell}^{\varepsilon}=\hat{v}_{\ell}^{\varepsilon}-\hat{V}_{\ell}$, which obviously satisfies

$$
\left\{\begin{aligned}
-\operatorname{div}\left(a\left(x_{\ell}, \frac{x}{\varepsilon}\right) \nabla \theta_{\ell}^{\varepsilon}(x)\right) & =0 & & x \in I_{\delta}\left(x_{\ell}\right), \\
\theta_{\ell}^{\varepsilon}(x) & =-\varepsilon \chi^{k}\left(x_{\ell}, \frac{x}{\varepsilon}\right) \frac{\partial V_{\ell}}{\partial x_{k}}(x) & & x \in \partial I_{\delta}\left(x_{\ell}\right) .
\end{aligned}\right.
$$


Note that $\theta_{\ell}^{\varepsilon}$ is simply the boundary layer correction for the cell problem (1.6) [5]. It is proved in [45, (1.51) in $\S 1.4]$, using the rescaling $x^{\prime}=x / \delta$ over $I_{\delta}$ and $\varepsilon^{\prime}=\varepsilon / \delta$.

$$
\left\|\nabla \theta_{\ell}^{\varepsilon}\right\|_{0, I_{\delta}} \leq C\left(\frac{\varepsilon}{\delta}\right)^{1 / 2}\left\|\nabla V_{\ell}\right\|_{0, I_{\delta}} .
$$

This together with (3.7) gives

$$
\left\|\nabla\left(v_{\ell}^{\varepsilon}-\hat{V}_{\ell}\right)\right\|_{0, I_{\delta}} \leq C\left(\left(\frac{\varepsilon}{\delta}\right)^{1 / 2}+\delta\right)\left\|\nabla V_{\ell}\right\|_{0, I_{\delta}} .
$$

A straightforward calculation gives

$$
\left\|\nabla \hat{V}_{\ell}\right\|_{0, I_{\delta} \backslash I_{\kappa \varepsilon}} \leq C\left(\frac{\varepsilon}{\delta}\right)^{1 / 2}\left\|\nabla V_{\ell}\right\|_{0, I_{\delta}}
$$

which together with (3.10) leads to

$$
\begin{aligned}
\left\|\nabla v_{\ell}^{\varepsilon}\right\|_{0, I_{\delta} \backslash I_{\kappa \varepsilon}} & \leq\left\|\nabla \hat{V}_{\ell}\right\|_{0, I_{\delta} \backslash I_{\kappa \varepsilon}}+\left\|\nabla\left(v_{\ell}^{\varepsilon}-\hat{V}_{\ell}\right)\right\|_{0, I_{\delta} \backslash I_{\kappa \varepsilon}} \\
& \leq\left\|\nabla \hat{V}_{\ell}\right\|_{0, I_{\delta} \backslash I_{\kappa \varepsilon}}+\left\|\nabla\left(v_{\ell}^{\varepsilon}-\hat{V}_{\ell}\right)\right\|_{0, I_{\delta}} \\
& \leq C\left(\left(\frac{\varepsilon}{\delta}\right)^{1 / 2}+\delta\right)\left\|\nabla V_{\ell}\right\|_{0, I_{\delta}} .
\end{aligned}
$$

This gives (3.6).

As in (3.6), we also have

$$
\left\|\nabla v_{\ell}^{\varepsilon}\right\|_{0, I_{\delta} \backslash I_{(\kappa-2) \varepsilon}} \leq C\left(\left(\frac{\varepsilon}{\delta}\right)^{1 / 2}+\delta\right)\left\|\nabla V_{\ell}\right\|_{0, I_{\delta}} .
$$

\section{Theorem 3.2.}

$$
e(\mathrm{HMM}) \leq C\left(\frac{\varepsilon}{\delta}+\delta\right) .
$$

Proof. Note that $v_{\ell}^{\varepsilon}=\left(v_{\ell}^{\varepsilon}-\hat{v}_{\ell}^{\varepsilon}\right)+\theta_{\ell}^{\varepsilon}+\hat{V}_{\ell}$. We have

$$
\left(\nabla W \cdot\left(\mathcal{A}_{H}-\mathcal{A}\right) \nabla V\right)\left(x_{\ell}\right)=: I_{1}+I_{2}+I_{3},
$$

where

$$
\begin{aligned}
& I_{1}=f_{I_{\delta}} \nabla w_{\ell}^{\varepsilon} \cdot a\left(x, \frac{x}{\varepsilon}\right) \nabla\left(v_{\ell}^{\varepsilon}-\hat{v}_{\ell}^{\varepsilon}\right) d x, \quad I_{2}=f_{I_{\delta}} \nabla w_{\ell}^{\varepsilon} \cdot a\left(x, \frac{x}{\varepsilon}\right) \nabla \theta_{\ell}^{\varepsilon} d x, \\
& I_{3}=f_{I_{\delta}} \nabla w_{\ell}^{\varepsilon} \cdot a\left(x, \frac{x}{\varepsilon}\right) \nabla \hat{V}_{\ell} d x-\nabla W_{\ell} \cdot \mathcal{A}\left(x_{\ell}\right) \nabla V_{\ell} .
\end{aligned}
$$

Using (3.7) and (2.2), we bound $I_{1}$ as

$$
\begin{aligned}
\left|I_{1}\right| & \leq \Lambda \delta^{-d}\left\|\nabla\left(v_{\ell}^{\varepsilon}-\hat{v}_{\ell}^{\varepsilon}\right)\right\|_{0, I_{\delta}}\left\|\nabla w_{\ell}^{\varepsilon}\right\|_{0, I_{\delta}} \\
& \leq C \delta^{1-d}\left\|\nabla V_{\ell}\right\|_{0, I_{\delta}}\left\|\nabla W_{\ell}\right\|_{0, I_{\delta}}=C \delta\left|\nabla V_{\ell}\right|\left|\nabla W_{\ell}\right| .
\end{aligned}
$$

Using the symmetry of $a^{\varepsilon}, I_{2}=f_{I_{\delta}} \nabla \theta_{\ell}^{\varepsilon} \cdot a\left(x, \frac{x}{\varepsilon}\right) \nabla w_{\ell}^{\varepsilon} d x$ and

$$
\begin{aligned}
I_{2}= & f_{I_{\delta}} \nabla\left(\theta_{\ell}^{\varepsilon}+\varepsilon \chi^{k}\left(x_{\ell}, \frac{x}{\varepsilon}\right) \frac{\partial V_{\ell}}{\partial x_{k}}\left(1-\rho^{\varepsilon}\right)\right) \cdot a\left(x, \frac{x}{\varepsilon}\right) \nabla w_{\ell}^{\varepsilon} d x \\
& -f_{I_{\delta}} \nabla\left(\varepsilon \chi^{k}\left(x_{\ell}, \frac{x}{\varepsilon}\right) \frac{\partial V_{\ell}}{\partial x_{k}}\left(1-\rho^{\varepsilon}\right)\right) \cdot a\left(x, \frac{x}{\varepsilon}\right) \nabla w_{\ell}^{\varepsilon} d x,
\end{aligned}
$$


where $\rho^{\varepsilon}(x) \in \mathcal{C}_{0}^{\infty}\left(I_{\delta}\right),\left|\nabla \rho^{\varepsilon}\right| \leq C / \varepsilon$, and

$$
\rho^{\varepsilon}(x)= \begin{cases}1 & \text { if } \operatorname{dist}\left(x, \partial I_{\delta}\right) \geq 2 \varepsilon \\ 0 & \text { if } \operatorname{dist}\left(x, \partial I_{\delta}\right) \leq \varepsilon\end{cases}
$$

Using (1.6) and $\theta_{\ell}^{\varepsilon}+\varepsilon \chi^{k}\left(x_{\ell}, \frac{x}{\varepsilon}\right) \frac{\partial V_{\ell}}{\partial x_{k}}\left(1-\rho^{\varepsilon}\right) \in H_{0}^{1}\left(I_{\delta}\right)$, integrating by parts makes the first term in the right-hand side of $I_{2}$ vanish; therefore we write $I_{2}$ as

$$
I_{2}=-f_{I_{\delta}} a_{i j}\left(x, \frac{x}{\varepsilon}\right) \frac{\partial w_{\ell}^{\varepsilon}}{\partial x_{i}} \frac{\partial \chi^{k}}{\partial y_{j}} \frac{\partial V_{\ell}}{\partial x_{k}}\left(1-\rho^{\varepsilon}\right) d x+\varepsilon f_{I_{\delta}} a_{i j}\left(x, \frac{x}{\varepsilon}\right) \frac{\partial w_{\ell}^{\varepsilon}}{\partial x_{i}} \chi^{k} \frac{\partial V_{\ell}}{\partial x_{k}} \frac{\partial \rho^{\varepsilon}}{\partial x_{j}} d x
$$

Using (3.12), we bound $I_{2}$ as

$$
\left|I_{2}\right| \leq C \delta^{-d}\left\|\nabla w_{\ell}^{\varepsilon}\right\|_{0, I_{\delta} \backslash I_{(\kappa-2) \varepsilon}}\left\|\nabla V_{\ell}\right\|_{0, I_{\delta} \backslash I_{(\kappa-2) \varepsilon}} \leq C\left(\frac{\varepsilon}{\delta}+\delta^{2}\right)\left|\nabla W_{\ell}\right|\left|\nabla V_{\ell}\right| .
$$

Using (3.2) and integrating by parts, we obtain

$$
f_{I_{\delta}} \nabla w_{\ell}^{\varepsilon} \cdot a\left(x_{\ell}, \frac{x}{\varepsilon}\right) \nabla \hat{V}_{\ell} d x=f_{I_{\delta}} \nabla W_{\ell} \cdot a\left(x_{\ell}, \frac{x}{\varepsilon}\right) \nabla \hat{V}_{\ell} d x
$$

which together with (3.5) gives

$$
\begin{aligned}
I_{3}= & f_{I_{\delta}} \nabla w_{\ell}^{\varepsilon}\left[a\left(x, \frac{x}{\varepsilon}\right)-a\left(x_{\ell}, \frac{x}{\varepsilon}\right)\right] \nabla \hat{V}_{\ell} d x \\
& +\frac{1}{\delta^{d}} \int_{I_{\delta} \backslash I_{\kappa \varepsilon}} \nabla W_{\ell} \cdot a\left(x_{\ell}, \frac{x}{\varepsilon}\right) \nabla \hat{V}_{\ell} d x+\left(|\kappa \varepsilon / \delta|^{d}-1\right) \nabla W_{\ell} \cdot \mathcal{A}\left(x_{\ell}\right) \nabla V_{\ell} .
\end{aligned}
$$

The last term of $I_{3}$ is bounded by

$$
|| \kappa \varepsilon /\left.\delta\right|^{d}-1|| \nabla W_{\ell} \cdot \mathcal{A}\left(x_{\ell}\right) \nabla V_{\ell}\left|\leq C \frac{\varepsilon}{\delta}\right| \nabla V_{\ell}|| \nabla W_{\ell} \mid,
$$

where we have used ||$\kappa \varepsilon /\left.\delta\right|^{d}-1 \mid \leq C \varepsilon / \delta$. Using (3.11), we get

$$
\begin{aligned}
\delta^{-d}\left|\int_{I_{\delta} \backslash I_{\kappa \varepsilon}} \nabla W_{\ell} \cdot a\left(x_{\ell}, \frac{x}{\varepsilon}\right) \nabla \hat{V}_{\ell} d x\right| & \leq C \frac{1}{\delta^{d}}\left\|\nabla \hat{V}_{\ell}\right\|_{0, I_{\delta} \backslash I_{\kappa \varepsilon}}\left\|\nabla W_{\ell}\right\|_{0, I_{\delta} \backslash I_{\kappa \varepsilon}} \\
& \leq C\left(\frac{\varepsilon}{\delta}+\delta^{2}\right)\left|\nabla V_{\ell} \| \nabla W_{\ell}\right| .
\end{aligned}
$$

Consequently, we obtain

$$
\begin{aligned}
\left|I_{3}\right| & \leq C \delta^{1-d}\left\|\nabla w_{\ell}^{\varepsilon}\right\|_{0, I_{\delta}}\left\|\nabla \hat{V}_{\ell}\right\|_{0, I_{\delta}}+C\left(\frac{\varepsilon}{\delta}+\delta^{2}\right)\left|\nabla V_{\ell}\right|\left|\nabla W_{\ell}\right| \\
& \leq C\left(\frac{\varepsilon}{\delta}+\delta\right)\left|\nabla V_{\ell} \| \nabla W_{\ell}\right| .
\end{aligned}
$$

Combining the estimates for $I_{1}, I_{2}$ and $I_{3}$ gives the desired result (3.13).

Remark 3.3. An explicit expression for $v_{\ell}^{\varepsilon}$ is available in one dimension, from which we may show that the bound for $e(\mathrm{HMM})$ is sharp. 


\section{Reconstruction AND COMPRESSION}

4.1. Reconstruction procedure. Next we consider how to construct better approximations to $u^{\varepsilon}$ from $U_{H}$. We will restrict ourselves to the case when $k=1$.

Proof of Theorem 1.4 Subtracting (1.1) from (1.14), we obtain

$$
\left\{\begin{aligned}
-\operatorname{div}\left(a^{\varepsilon}(x) \nabla\left(\tilde{u}^{\varepsilon}-u^{\varepsilon}\right)(x)\right) & =0 & & x \in \Omega_{\eta}, \\
\left(\tilde{u}^{\varepsilon}-u^{\varepsilon}\right)(x) & =U_{H}(x)-u^{\varepsilon}(x) & & x \in \partial \Omega_{\eta} .
\end{aligned}\right.
$$

Using classical interior estimates for elliptic equation [24], we have

$$
\left\|\nabla\left(\tilde{u}^{\varepsilon}-u^{\varepsilon}\right)\right\|_{0, \Omega} \leq \frac{C}{\eta}\left\|\tilde{u}^{\varepsilon}-u^{\varepsilon}\right\|_{0, \Omega_{\eta}} .
$$

Using the Hopf maximum principle, we get

$$
\begin{aligned}
\frac{1}{\eta^{2}} \int_{\Omega_{\eta}}\left|\left(\tilde{u}^{\varepsilon}-u^{\varepsilon}\right)(x)\right|^{2} d x & \leq \frac{C}{\eta^{2}}\left\|\tilde{u}^{\varepsilon}-u^{\varepsilon}\right\|_{L^{\infty}\left(\Omega_{\eta}\right)}^{2} \leq \frac{C}{\eta^{2}}\left\|u^{\varepsilon}-U_{H}\right\|_{L^{\infty}\left(\partial \Omega_{\eta}\right)}^{2} \\
& \leq \frac{C}{\eta^{2}}\left(\left\|U_{0}-U_{H}\right\|_{L^{\infty}\left(\Omega_{\eta}\right)}^{2}+\left\|u^{\varepsilon}-U_{0}\right\|_{L^{\infty}\left(\Omega_{\eta}\right)}^{2}\right) .
\end{aligned}
$$

A combination of the above two results implies Theorem 1.4.

Proof of Theorem 1.5. Denote $I_{\varepsilon}\left(x_{K}\right)=x_{K}+\varepsilon I$ and define $\hat{u}^{\varepsilon}$ as the solution of

$$
-\operatorname{div}\left(a\left(x_{K}, \frac{x}{\varepsilon}\right) \nabla \hat{u}^{\varepsilon}(x)\right)=0 \quad \text { in } I_{\varepsilon}\left(x_{K}\right),
$$

with the boundary condition that $\hat{u}^{\varepsilon}-U_{H}$ is periodic on $\partial I_{\varepsilon}\left(x_{K}\right)$ and

$$
\int_{I_{\varepsilon}\left(x_{K}\right)}\left(\hat{u}^{\varepsilon}-U_{H}\right) d x=0,
$$

where $x_{K}$ is the barycenter of $K$.

It is easy to verify that $\hat{u}^{\varepsilon}$ takes the explicit form

$$
\hat{u}^{\varepsilon}(x)=U_{H}(x)+\varepsilon \chi^{k}\left(x_{K}, \frac{x}{\varepsilon}\right) \frac{\partial U_{H}}{\partial x_{k}}(x) .
$$

Note that the periodic extension of $\hat{u}^{\varepsilon}-U_{H}$ is still $\varepsilon \chi^{k}\left(x_{K}, \frac{x}{\varepsilon}\right) \frac{\partial U_{H}}{\partial x_{k}}(x)$. This means that $\hat{u}^{\varepsilon}$ is also well defined for the whole of $K$ and takes the same explicit form as (4.2).

Using $\int_{I} \chi^{k}\left(x_{K}, y\right) d y=0$ for $k=1, \cdots, d$ and that $\nabla U_{H}$ is a piecewise constant on $K$, we obtain

$$
\int_{I_{\varepsilon}\left(x_{K}\right)}\left(\hat{u}^{\varepsilon}-U_{H}\right)(x) d x=\int_{I_{\varepsilon}\left(x_{K}\right)} \varepsilon \chi^{k}\left(x_{K}, \frac{x}{\varepsilon}\right) \frac{\partial U_{H}}{\partial x_{k}}(x) d x=0 .
$$

As in (3.7), we have

$$
\left\|\nabla\left(\tilde{u}^{\varepsilon}-\hat{u}^{\varepsilon}\right)\right\|_{0, I_{\varepsilon}\left(x_{K}\right)} \leq C \varepsilon\left\|\nabla U_{H}\right\|_{0, I_{\varepsilon}\left(x_{K}\right)} .
$$

From the construction of $\tilde{u}^{\varepsilon}$, we have for any $x_{1} \in K$,

$$
\left\|\nabla\left(\tilde{u}^{\varepsilon}-\hat{u}^{\varepsilon}\right)\right\|_{0, I_{\varepsilon}\left(x_{1}\right)}=\left\|\nabla\left(\tilde{u}^{\varepsilon}-\hat{u}^{\varepsilon}\right)\right\|_{0, I_{\varepsilon}\left(x_{K}\right)} .
$$

Since $\nabla U_{H}$ is constant over $K$, we get

$$
\left\|\nabla\left(\tilde{u}^{\varepsilon}-\hat{u}^{\varepsilon}\right)\right\|_{0, K} \leq C \varepsilon\left\|\nabla U_{H}\right\|_{0, K} .
$$


Adding up for all $K \in \mathcal{T}_{H}$ and using the a priori estimate $\left\|\nabla U_{H}\right\|_{0} \leq C\|f\|_{0}$, we obtain

$$
\left(\sum_{K \in \mathcal{T}_{H}}\left\|\nabla\left(\tilde{u}^{\varepsilon}-\hat{u}^{\varepsilon}\right)\right\|_{0, K}^{2}\right)^{1 / 2} \leq C \varepsilon\left\|\nabla U_{H}\right\|_{0} \leq C \varepsilon .
$$

Using (4.2), a straightforward calculation gives

$$
\frac{\partial \hat{u}^{\varepsilon}}{\partial x_{i}}=\frac{\partial U_{H}}{\partial x_{i}}+\frac{\partial \chi^{k}}{\partial y_{i}}\left(x_{K}, \frac{x}{\varepsilon}\right) \frac{\partial U_{H}}{\partial x_{k}} .
$$

Define the first order approximation of $u^{\varepsilon}$ as

$$
u_{1}^{\varepsilon}(x)=U_{0}+\varepsilon \chi^{k}\left(x, \frac{x}{\varepsilon}\right) \frac{\partial U_{0}}{\partial x_{k}},
$$

where $\left\{\chi^{k}\right\}_{k=1}^{d}$ is the solutions of (3.2). Obviously,

$$
\frac{\partial u_{1}^{\varepsilon}}{\partial x_{i}}=\frac{\partial U_{0}}{\partial x_{i}}+\left(\varepsilon \frac{\partial \chi^{k}}{\partial x_{i}}+\frac{\partial \chi^{k}}{\partial y_{i}}\right)\left(x, \frac{x}{\varepsilon}\right) \frac{\partial U_{0}}{\partial x_{i}}+\varepsilon \chi^{k}\left(x, \frac{x}{\varepsilon}\right) \frac{\partial^{2} U_{0}}{\partial x_{i} \partial x_{k}} .
$$

A combination of the above estimates leads to

$$
\begin{aligned}
\left\|\nabla\left(\hat{u}^{\varepsilon}-u_{1}^{\varepsilon}\right)\right\|_{0, K} & \leq C\left\|\nabla\left(U_{H}-U_{0}\right)\right\|_{0, K}+C \varepsilon\left|U_{0}\right|_{1, K} \\
& +\left\|\left(\frac{\partial \chi^{k}}{\partial y_{i}}\left(x, \frac{x}{\varepsilon}\right)-\frac{\partial \chi^{k}}{\partial y_{i}}\left(x_{K}, \frac{x}{\varepsilon}\right)\right) \frac{\partial U_{0}}{\partial x_{k}}\right\|_{0, K}+C \varepsilon\left|U_{0}\right|_{2, K} \\
& \leq C\left|U_{0}-U_{H}\right|_{1, K}+C(\varepsilon+H)\left\|U_{0}\right\|_{2, K} .
\end{aligned}
$$

Summing up for all $K \in \mathcal{T}_{H}$ and using Theorem 1.1 for $k=1$ and Theorem 1.2 for the case $I_{\delta}=x_{K}+\varepsilon I$, we get

$$
\left(\sum_{K \in \mathcal{T}_{H}}\left\|\nabla\left(\hat{u}^{\varepsilon}-u_{1}^{\varepsilon}\right)\right\|_{0, K}^{2}\right)^{1 / 2} \leq C(\varepsilon+H),
$$

which together with (4.5) and the classical estimate for $u^{\varepsilon}-u_{1}^{\varepsilon}$ [5, 32, 45, i.e.,

$$
\left\|u^{\varepsilon}-u_{1}^{\varepsilon}\right\|_{1} \leq C \sqrt{\varepsilon}
$$

gives

$$
\begin{aligned}
\left(\sum_{K \in \mathcal{T}_{H}}\left\|\nabla\left(u^{\varepsilon}-\tilde{u}^{\varepsilon}\right)\right\|_{0, K}^{2}\right)^{1 / 2} \leq & \left\|u^{\varepsilon}-u_{1}^{\varepsilon}\right\|_{1}+\left(\sum_{K \in \mathcal{T}_{H}}\left\|\nabla\left(u_{1}^{\varepsilon}-\hat{u}^{\varepsilon}\right)\right\|_{0, K}^{2}\right)^{1 / 2} \\
& +\left(\sum_{K \in \mathcal{T}_{H}}\left\|\nabla\left(\hat{u}^{\varepsilon}-\tilde{u}^{\varepsilon}\right)\right\|_{0, K}^{2}\right)^{1 / 2} \\
\leq & C(\sqrt{\varepsilon}+H) .
\end{aligned}
$$

\section{Corollary 4.1.}

$$
\left\|\tilde{u}^{\varepsilon}-u^{\varepsilon}\right\|_{0} \leq C\left(\varepsilon+H^{2}\right) .
$$

Proof. Using the definition of $\tilde{u}^{\varepsilon}$, we have $\int_{I_{\varepsilon}\left(x_{K}\right)}\left(\tilde{u}^{\varepsilon}-U_{H}\right)(x) d x=0$. Together with 4.31), we have

$$
\int_{I_{\varepsilon}\left(x_{K}\right)}\left(\tilde{u}^{\varepsilon}-\hat{u}^{\varepsilon}\right)(x) d x=0 .
$$


An application of the Poincaré inequality gives

$$
\left\|\tilde{u}^{\varepsilon}-\hat{u}^{\varepsilon}\right\|_{0, I_{\varepsilon}\left(x_{K}\right)} \leq C \varepsilon\left\|\nabla\left(\tilde{u}^{\varepsilon}-\hat{u}^{\varepsilon}\right)\right\|_{0, I_{\varepsilon}\left(x_{K}\right)} \leq C \varepsilon^{2}\left\|\nabla U_{H}\right\|_{0, I_{\varepsilon}\left(x_{K}\right)} .
$$

As before for any $I_{\varepsilon}\left(x_{1}\right),\left\|\tilde{u}^{\varepsilon}-\hat{u}^{\varepsilon}\right\|_{0, I_{\varepsilon}\left(x_{1}\right)}=\left\|\tilde{u}^{\varepsilon}-\hat{u}^{\varepsilon}\right\|_{0, I_{\varepsilon}\left(x_{K}\right)}$, note that $\nabla U_{H}$ is a constant on $K$. We obtain

$$
\left\|\tilde{u}^{\varepsilon}-\hat{u}^{\varepsilon}\right\|_{0, K} \leq C \varepsilon^{2}\left\|\nabla U_{H}\right\|_{0, K} .
$$

On each element $K$, we have

$$
\left\|\hat{u}^{\varepsilon}-U_{H}\right\|_{0, K} \leq C \varepsilon\left\|\nabla U_{H}\right\|_{0, K} .
$$

Combining the above and summing up for all $K \in \mathcal{T}_{H}$, we get

$$
\left\|\tilde{u}^{\varepsilon}-U_{H}\right\|_{0} \leq C \varepsilon\left\|\nabla U_{H}\right\|_{0} \leq C \varepsilon
$$

which together with

$$
\left\|u^{\varepsilon}-U_{H}\right\|_{0} \leq\left\|u^{\varepsilon}-U_{0}\right\|_{0}+\left\|U_{0}-U_{H}\right\|_{0} \leq C\left(\varepsilon+H^{2}\right)
$$

leads to (4.6), where we have used the estimate for $U_{0}$ [5, 32, 45], i.e.,

$$
\left\|u^{\varepsilon}-U_{0}\right\|_{0} \leq C \varepsilon \text {. }
$$

4.2. Compression operator. The compression operator (denoted by $Q$ ) maps the microvariables to the macrovariables [16]. It plays an important role in the general framework of HMM, even though for the present problem HMM can be formulated without explicitly specifying the compression operator beforehand. Typically the compression operator is some spatial/temporal averaging, or projection to some slow manifolds. It is of interest to consider the error bound for $Q u^{\varepsilon}-U_{H}$. We first list some natural properties of the compression operator.

- For any $\phi \in X, Q \phi \in X_{H}$.

- There exists a constant $C$ such that

$$
\|Q \phi\|_{0} \leq C\|\phi\|_{0} \text {. }
$$

- For any $k \geq 1$, if $\phi \in H^{k+1}(\Omega) \cap H_{0}^{1}(\Omega)$, then

$$
\|\phi-Q \phi\|_{0} \leq C H^{k+1}|\phi|_{k+1} .
$$

Theorem 4.2. Assume that $Q$ satisfies all three requirements and $U_{0} \in H^{k+1}(D)$ for any $k \geq 1$. Then

$$
\left\|Q u^{\varepsilon}-U_{H}\right\|_{0} \leq C\left(\varepsilon+H^{k+1}\right) .
$$

Moreover, if $\mathcal{T}_{H}$ is quasi-uniform, then

$$
\left\|Q u^{\varepsilon}-U_{H}\right\|_{1} \leq C\left(\frac{\varepsilon}{H}+H^{k}\right) .
$$

Proof. We decompose $Q u^{\varepsilon}-U_{H}$ into

$$
Q u^{\varepsilon}-U_{H}=Q\left(u^{\varepsilon}-U_{0}\right)+\left(Q U_{0}-U_{0}\right)+\left(U_{0}-U_{H}\right) .
$$

Using the fact that $Q$ is bounded in $\mathrm{L}_{\text {norm }}^{2}$, we obtain

$$
\left\|Q\left(u^{\varepsilon}-U_{0}\right)\right\|_{0} \leq C\left\|u^{\varepsilon}-U_{0}\right\|_{0} \leq C \varepsilon .
$$

Using the third property of $Q$, we have

$$
\left\|Q U_{0}-U_{0}\right\|_{0} \leq C H^{k+1} .
$$

Using Theorem 1.1 and the first estimate in Theorem 1.2, we have

$$
\left\|U_{0}-U_{H}\right\|_{0} \leq C\left(\varepsilon+H^{k+1}\right) .
$$


A combination of these three estimates implies (4.8), which together with the inverse inequality (cf. Theorem 1.7) leads to (4.9).

It remains to give some examples of the compression operator. The following two types of operators meet all three requirements:

- the $\mathrm{L}^{2}$-projection operator onto $X_{H}$,

- the Clément-type interpolation operator [12].

Remark 4.3. Notice that in one dimension, the standard Lagrange interpolant does not meet the second requirement. However, it is still possible to derive (4.9) via another approach. Moreover, a careful study of one dimensional examples shows that the term $\varepsilon / H$ in 4.9 is sharp.

\section{Nonlinear homogenization PROBlems}

5.1. Algorithms and main results. We consider the following nonlinear problem which has been discussed in [6, 23]:

$$
\left\{\begin{aligned}
-\operatorname{div}\left(a^{\varepsilon}\left(x, u^{\varepsilon}(x)\right) \nabla u^{\varepsilon}(x)\right) & =f(x) & & x \in D, \\
u^{\varepsilon}(x) & =0 & & x \in \partial D .
\end{aligned}\right.
$$

In this section, we define $X:=W_{0}^{1, p}(D)$ with $p>1$ and $X_{H}$ is defined as the $\mathcal{P}_{k}$ finite element subspace of $X$.

We assume that $a^{\varepsilon}\left(x, u^{\varepsilon}\right)$ satisfies

$$
\lambda|\xi|^{2} \leq a_{i j}^{\varepsilon} \xi_{i} \xi_{j} \leq \Lambda|\xi|^{2} \quad \text { for all } \xi \in \mathbb{R}^{d},
$$

with $0<\lambda \leq \Lambda$. Moreover, we assume that $a^{\varepsilon}(x, z)$ is equi-continuous in $z$ uniformly with respect to $x$ and $\varepsilon$.

The homogenized problem, if it exists, is of the following form:

$$
\left\{\begin{aligned}
\mathcal{L} U_{0}:=-\operatorname{div}\left(\mathcal{A}\left(x, U_{0}(x)\right) \nabla U_{0}(x)\right) & =f(x) & & x \in D, \\
U_{0}(x) & =0 & & x \in \partial D .
\end{aligned}\right.
$$

If we let

$$
A(v, w)=(\mathcal{A}(x, v) \nabla v, \nabla w) \quad \text { for all } v, w \in X,
$$

then

$$
A\left(U_{0}, v\right)=(f, v) \text { for all } v \in X^{\prime},
$$

where $X^{\prime}$ is the dual space of $X$.

The linearized operator of $\mathcal{L}$ at $U_{0}$ is defined for any $v \in H_{0}^{1}(D)$ by

$$
\mathcal{L}_{\operatorname{lin}}\left(U_{0}\right) v=-\operatorname{div}\left(\mathcal{A}\left(x, U_{0}\right) \nabla v+\mathcal{A}_{p}\left(x, U_{0}\right) \nabla U_{0} v\right),
$$

where $\mathcal{A}_{p}(x, z):=\nabla_{z} \mathcal{A}(x, z) . \mathcal{L}_{\text {lin }}$ induces a bilinear form through

$$
\hat{A}(u ; v, w):=(\mathcal{A}(x, u) \nabla v, \nabla w)+\left(\mathcal{A}_{p}(x, u) \nabla u v, \nabla w\right) \quad \text { for all } v, w \in H_{0}^{1}(D) .
$$

Our basic assumption is that the linearized operator $\mathcal{L}_{\text {lin }}$ is an isomorphism from $H_{0}^{1}(D)$ to $H^{-1}(D)$, so $U_{0}$ must be an isolated solution of (5.2). 
To formulate HMM, for each quadrature point $x_{\ell}$, define $v_{\ell}^{\varepsilon}$ to be the solutions of

$$
\left\{\begin{aligned}
-\operatorname{div}\left(a^{\varepsilon}\left(x, v_{\ell}^{\varepsilon}\right) \nabla v_{\ell}^{\varepsilon}(x)\right) & =0 & & x \in I_{\delta}\left(x_{\ell}\right), \\
v_{\ell}^{\varepsilon}(x) & =V_{\ell}(x) & & x \in \partial I_{\delta}\left(x_{\ell}\right) .
\end{aligned}\right.
$$

We can define $w_{\ell}^{\varepsilon}$ similarly.

For any $V, W \in X_{H}$, define

$$
\nabla W\left(x_{\ell}\right) \cdot \mathcal{A}_{H}\left(x_{\ell}, V\left(x_{\ell}\right)\right) \nabla V\left(x_{\ell}\right)=f_{I_{\delta}\left(x_{\ell}\right)} \nabla w_{\ell}^{\varepsilon}(x) \cdot a^{\varepsilon}\left(x, v_{\ell}^{\varepsilon}(x)\right) \nabla v_{\ell}^{\varepsilon}(x) d x
$$

and

$$
A_{H}(V, W):=\sum_{K \in \mathcal{T}_{H}}|K| \sum_{x_{\ell} \in K} \omega_{\ell} \nabla W\left(x_{\ell}\right) \cdot \mathcal{A}_{H}\left(x_{\ell}, V\left(x_{\ell}\right)\right) \nabla V\left(x_{\ell}\right) .
$$

The HMM solution is given by the problem:

Problem 5.1. Find $U_{H} \in X_{H}$ such that

$$
A_{H}\left(U_{H}, V\right)=(f, V) \text { for all } V \in X_{H} .
$$

For any $v, v_{H}, w \in X$, define

$$
R\left(v, v_{H}, w\right):=A\left(v_{H}, w\right)-A(v, w)-\hat{A}\left(v ; v_{H}-v, w\right) .
$$

It is easy to see that for any $v$ and $v_{H}$ satisfying $\|v\|_{1, \infty}+\left\|v_{H}\right\|_{1, \infty} \leq M$,

$$
\left|R\left(v, v_{H}, w\right)\right| \leq C(M)\left(\left\|e_{H}\right\|_{0,2 p}^{2}+\left\|e_{H} \nabla e_{H}\right\|_{0, p}\right)\|\nabla w\|_{0, q}
$$

for $e_{H}:=v-v_{H}$ and $\frac{1}{p}+\frac{1}{q}=1, p, q \geq 1$ (see [42, Lemma 3.1] for a similar result). Therefore we have

Lemma 5.2. $U_{H} \in X_{H}$ is the solution of Problem 5.1 if and only if

$$
\begin{aligned}
\hat{A}\left(U_{0} ; U_{0}-U_{H}, V\right)= & R\left(U_{0}, U_{H}, V\right) \\
& +A_{H}\left(U_{H}, V\right)-A\left(U_{H}, V\right) \quad \text { for all } V \in X_{H} .
\end{aligned}
$$

For any $V, W \in X_{H}$, define

$$
E(V, W):=\nabla W\left(x_{\ell}\right) \cdot\left(\mathcal{A}_{H}-\mathcal{A}\right)\left(x_{\ell}, V\left(x_{\ell}\right)\right) \nabla V\left(x_{\ell}\right) .
$$

Define $e(\mathrm{HMM})$ as

$$
e(\mathrm{HMM})=\max _{\substack{x_{\ell} \in K, K \in \mathcal{T}_{H} \\ V \in X_{H} \cap W^{1, \infty}(D), W \in X_{H}}} \frac{|E(V, W)|}{\left|\nabla V_{\ell}\right|\left|\nabla W_{\ell}\right|} .
$$

The existence and uniqueness of the solution of Problem 5.1 are proved in the following lemma.

Lemma 5.3. Assume that $U_{0} \in W^{2, p}(D)$ with $p>d$ and $\mathcal{L}_{\text {lin }}$ is an isomorphism from $H_{0}^{1}(D)$ to $H^{-1}(D)$. If $e(\mathrm{HMM})$ is uniformly bounded and there exist constants $H_{0}>0$ and $\mathcal{M}_{1}>0$ such that for $0<H \leq H_{0}$ and

$$
e(\mathrm{HMM})^{1 / 2}|\ln H| \leq \mathcal{M}_{1},
$$

then Problem 5.1 has a solution $U_{H}$ satisfying

$$
\begin{aligned}
\left\|U_{H}-P_{H} U_{0}\right\|_{1, \infty} & \leq e(\mathrm{HMM})^{1 / 2}+H^{1-d / p} \\
\left\|U_{0}-U_{H}\right\|_{1, \infty} & \leq C\left(e(\mathrm{HMM})^{1 / 2}+H^{1-d / p}\right),
\end{aligned}
$$


where $P_{H} U_{0} \in X_{H}$ is defined as

$$
\hat{A}\left(U_{0} ; P_{H} U_{0}, V\right)=\hat{A}\left(U_{0} ; U_{0}, V\right) \text { for all } V \in X_{H} .
$$

Moreover, if there exists a constant $\eta(M)$ with $0<\eta(M)<1$ such that

$$
\sum_{K \in \mathcal{T}_{H}}|K| \sum_{x_{\ell} \in K} \omega_{\ell}|E(V, Z)-E(W, Z)| \leq \eta(M)\|V-W\|_{1}\|Z\|_{1}
$$

for all $V, W \in X_{H} \cap W^{1, \infty}(D)$ and $Z \in X_{H}$, satisfying $\|V\|_{1, \infty}+\|W\|_{1, \infty} \leq M$, then there exists a constant $H_{1}>0$ such that for $0<H \leq H_{1}$, the HMM solution $U_{H}$ satisfying (5.11) $)_{1}$ is locally unique.

Proof. Since $\mathcal{L}_{\text {lin }}$ is an isomorphism from $H_{0}^{1}(D)$ to $H^{-1}(D)$, there exists a constant $C$ such that

$$
\sup _{W \in H_{0}^{1}(D)} \frac{\hat{A}\left(U_{0} ; V, W\right)}{\|W\|_{1}} \geq C\|V\|_{1} \quad \text { for all } V \in H_{0}^{1}(D) .
$$

Using [42] Lemma 2.2], we conclude that there exists a constant $H_{2}>0$ such that for $0<H \leq H_{2}$,

$$
\sup _{W \in X_{H}} \frac{\hat{A}\left(U_{0} ; V, W\right)}{\|W\|_{1}} \geq C\|V\|_{1} \quad \text { for all } V \in X_{H} .
$$

Therefore there is a unique solution $P_{H} U_{0} \in X_{H}$ satisfying (5.12) and

$$
\left\|U_{0}-P_{H} U_{0}\right\|_{1, \infty} \leq C H^{1-d / p} .
$$

Moreover, let $\hat{G}_{H}^{z}$ be the finite element approximation of the regularized Green's function associated with $\hat{A}\left(U_{0} ; \cdot, \cdot\right)$. Using [42, equation 2.11], or using (5.14), similarly to (2.11), we have

$$
\left\|\hat{G}_{H}^{z}\right\|_{1,1} \leq C|\ln H|
$$

Define a nonlinear mapping $T: X_{H} \rightarrow X_{H}$ by

$$
\hat{A}\left(U_{0} ; T(V), W\right)=\hat{A}\left(U_{0} ; U_{0}, W\right)-R\left(U_{0}, V, W\right)+A(V, W)-A_{H}(V, W),
$$

for any $W \in X_{H}$. Obviously $T$ is continuous due to (5.14) and (5.6).

Let

$$
B:=\left\{V \in X_{H} \mid\left\|V-P_{H} U_{0}\right\|_{1, \infty} \leq e(\mathrm{HMM})^{1 / 2}+H^{1-d / p}\right\} .
$$

We next prove that there exists a constant $H_{0}>0$ such that for all $0<H \leq H_{0}$, $T(B) \subset B$.

Notice that

$$
\hat{A}\left(U_{0} ; T(V)-P_{H} U_{0}, W\right)=-R\left(U_{0}, V, W\right)+A(V, W)-A_{H}(V, W) .
$$

Taking $W=\hat{G}_{H}^{z}$ in the above equation, using (5.16) and (5.6), we obtain, for $\|V\|_{1, \infty} \leq M$,

$$
\begin{aligned}
& \left\|T(V)-P_{H} U_{0}\right\|_{1, \infty} \leq C(M)\left\|U_{0}-V\right\|_{1, \infty}^{2}|\ln H|+C(e(\mathrm{HMM})+H)|\ln H|\|V\|_{1, \infty} \\
& \leq C(M)\left(\left\|U_{0}-P_{H} U_{0}\right\|_{1, \infty}^{2}+\left\|P_{H} U_{0}-V\right\|_{1, \infty}^{2}\right)|\ln H|+C M(e(\mathrm{HMM})+H)|\ln H| \\
& \leq C(M)\left(e(\mathrm{HMM})+H^{2-2 d / p}\right)|\ln H|+C M(e(\mathrm{HMM})+H)|\ln H| .
\end{aligned}
$$


Since $V \in B$ and $e(\mathrm{HMM})$ is uniformly bounded, e.g., $e(\mathrm{HMM}) \leq M_{1}$, we have

$$
\begin{aligned}
\|V\|_{1, \infty} & \leq\left\|V-P_{H} U_{0}\right\|_{1, \infty}+\left\|P_{H} U_{0}\right\|_{1, \infty} \leq C\left(U_{0}\right)+e(\mathrm{HMM})^{1 / 2} \\
& \leq C\left(U_{0}\right)+M_{1}^{1 / 2}=: M_{0} .
\end{aligned}
$$

Combining the above two estimates, we obtain

$$
\left\|T(V)-P_{H} U_{0}\right\|_{1, \infty} \leq C\left(M_{0}\right)\left(e(\mathrm{HMM})+H^{2-2 d / p}+H\right)|\ln H| .
$$

Define $\mathcal{M}_{1}:=1 / C\left(M_{0}\right)$. Using (5.10), we obtain

$$
\left\|T(V)-P_{H} U_{0}\right\|_{1, \infty} \leq e(\mathrm{HMM})^{1 / 2}+C\left(M_{0}\right)\left(H^{2-2 d / p}+H\right)|\ln H| .
$$

Therefore there exits a constant $H_{3}$ such that for $0<H \leq H_{3}$, we have

$$
\left\|T(V)-P_{H} U_{0}\right\|_{1, \infty} \leq e(\mathrm{HMM})^{1 / 2}+H^{1-d / p} .
$$

Let $H_{0}:=\min \left(H_{2}, H_{3}\right)$. Then for $0<H \leq H_{0}$, we have $T(B) \subset B$. An application of Brouwer's fixed point theorem gives the existence of a $U_{H} \in B$ such that $T\left(U_{H}\right)=$ $U_{H}$. By definition, $U_{H}$ satisfies (5.11) $)_{1}$. Together with (5.15) it yields $(5.11)_{2}$.

To prove uniqueness, assume that both $U_{H}$ and $\hat{U}_{H}$ are solutions of (5.4) satisfying (5.11) 1 . Using (5.14), we obtain

$$
\begin{aligned}
C\left\|U_{H}-\hat{U}_{H}\right\|_{1} & \leq \sup _{W \in X_{H}} \frac{\int_{0}^{1} \hat{A}\left(U_{H}^{t} ; U_{H}-\hat{U}_{H}, W\right) d t}{\|W\|_{1}} \\
& \leq \sup _{W \in X_{H}} \frac{\left|A\left(U_{H}, W\right)-A\left(\hat{U}_{H}, W\right)\right|}{\|W\|_{1}}
\end{aligned}
$$

where $U_{H}^{t}=(1-t) \hat{U}_{H}+t U_{H}$. Note that

$$
\begin{aligned}
A\left(U_{H}, W\right)-A\left(\hat{U}_{H}, W\right) & =\left(A\left(U_{H}, W\right)-A_{H}\left(U_{H}, W\right)\right) \\
& -\left(A\left(\hat{U}_{H}, W\right)-A_{H}\left(\hat{U}_{H}, W\right)\right) .
\end{aligned}
$$

Since both $U_{H}$ and $\hat{U}_{H}$ sit in the set $B$, we can use (5.17) to get $\left\|U_{H}\right\|_{1, \infty}+$ $\left\|\hat{U}_{H}\right\|_{1, \infty} \leq 2 M_{0}$. Using (5.13), we obtain

$$
\left\|U_{H}-\hat{U}_{H}\right\|_{1} \leq\left(\eta\left(2 M_{0}\right)+C_{1} H\right)\left\|U_{H}-\hat{U}_{H}\right\|_{1} .
$$

If we choose $H_{1}$ such that

$$
\eta\left(2 M_{0}\right)+C_{1} H_{1}<1,
$$

then if $H<H_{1}$, we have $U_{H}=\hat{U}_{H}$. Therefore the HMM solution is locally unique.

From here on, when we talk about the HMM solution, we are referring to this particular solution that satisfies the condition in Lemma 5.3.

Based on the above lemma, we prove a nonlinear analog of Theorem 1.1.

Theorem 5.4. Under the assumptions in Lemma 5.3, let $U_{0}$ and $U_{H}$ be solutions of (5.2) and (5.4), respectively. Assume in addition that $U_{0} \in W^{k+1, \infty}(D)$. Then there exist constants $H_{0}$ and $\mathcal{M}_{1}^{*}$ such that if $0<H<H_{0}$ and $\mathcal{M}_{1}<\mathcal{M}_{1}^{*}$, then

$$
\begin{aligned}
\left\|U_{0}-U_{H}\right\|_{1} & \leq C\left(H^{k}+e(\mathrm{HMM})\right), \\
\left\|U_{0}-U_{H}\right\|_{1, \infty} & \leq C\left(H^{k}+e(\mathrm{HMM})\right)|\ln H| .
\end{aligned}
$$


Proof. Note that $U_{0} \in W^{k+1, \infty}(D)$ and from (5.14), we have

$$
\left\|U_{0}-P_{H} U_{0}\right\|_{1} \leq C H^{k}, \quad\left\|U_{0}-P_{H} U_{0}\right\|_{1, \infty} \leq C H^{k} .
$$

Using (5.7) with $V=P_{H} U_{0}-U_{H}$ and (5.14) and (2.3), we obtain

$$
\left\|P_{H} U_{0}-U_{H}\right\|_{1} \leq C\left\|U_{0}-U_{H}\right\|_{1,4}^{2}+C\left(H^{k}+e(\mathrm{HMM})\right) .
$$

Using the interpolation inequality

$$
\left\|U_{0}-U_{H}\right\|_{1,4}^{2} \leq\left\|U_{0}-U_{H}\right\|_{1}\left\|U_{0}-U_{H}\right\|_{1, \infty}
$$

together with (5.20) and (5.17) gives

$$
\begin{aligned}
\left\|P_{H} U_{0}-U_{H}\right\|_{1} \leq & C_{1}\left(e(\mathrm{HMM})^{1 / 2}+H^{1-d / p}\right)\left\|P_{H} U_{0}-U_{H}\right\|_{1} \\
& +C\left(H^{k}+e(\mathrm{HMM})\right) .
\end{aligned}
$$

Let $V=\hat{G}_{H}^{z}$ in (5.7). Using (5.16), we obtain

$$
\begin{aligned}
\left\|P_{H} U_{0}-U_{H}\right\|_{1, \infty} \leq & C_{2}(e(\mathrm{HMM})+H)|\ln H|\left\|P_{H} U_{0}-U_{H}\right\|_{1, \infty} \\
& +C\left(\left\|U_{0}-U_{H}\right\|_{1, \infty}^{2}+e(\mathrm{HMM})+H^{k}\right)|\ln H| .
\end{aligned}
$$

Since (5.10) holds, using (5.11) 2 and $(5.20)_{2}$, we obtain

$$
\begin{aligned}
\left\|P_{H} U_{0}-U_{H}\right\|_{1, \infty} \leq & C_{2}\left(e(\mathrm{HMM})^{1 / 2}+H^{1-d / p}\right)|\ln H|\left\|P_{H} U_{0}-U_{H}\right\|_{1, \infty} \\
& +C\left(e(\mathrm{HMM})+H^{k}\right)|\ln H| .
\end{aligned}
$$

Now we choose

$$
\mathcal{M}_{1}^{*}:=\min \left(\frac{|\ln H|}{2 C_{1}}, \frac{1}{2 C_{2}}\right)
$$

and $H_{0}$ such that $e(\mathrm{HMM})^{1 / 2}|\ln H| \leq M_{1}^{*}$, and therefore

$$
\begin{aligned}
C_{1}\left(e(\mathrm{HMM})^{1 / 2}+H^{1-d / p}\right) & \leq \frac{1}{2}+C_{1} H_{0}^{1-d / p}<1, \\
C_{2}\left(e(\mathrm{HMM})^{1 / 2}+H^{1-d / p}\right)|\ln H| & \leq \frac{1}{2}+C_{2} H^{1-d / p}|\ln H|<1 .
\end{aligned}
$$

Thus we obtain

$$
\begin{aligned}
\left\|P_{H} U_{0}-U_{H}\right\|_{1} & \leq C\left(H^{k}+e(\mathrm{HMM})\right), \\
\left\|P_{H} U_{0}-U_{H}\right\|_{1, \infty} & \leq C\left(H^{k}+e(\mathrm{HMM})\right)|\ln H| .
\end{aligned}
$$

Using (5.20) once again gives (5.18) and (5.19).

5.2. Estimating $e(\mathrm{HMM})$. It remains to estimate $e(\mathrm{HMM})$ and verify assumptions (5.10) and (5.13). We assume that $a^{\varepsilon}\left(x, u^{\varepsilon}\right)=\left(a_{i j}\left(x, x / \varepsilon, u^{\varepsilon}\right)\right)$, and for $1 \leq i, j \leq d$, the coefficients $a_{i j}^{\varepsilon}(x, y, z)$ are smooth in $x, z$ and periodic in $y$ with period $I$. These types of problems, among others, have been considered in [5, 6, 23]. The homogenized coefficient $\mathcal{A}=\left(\mathcal{A}_{i j}(x, p)\right)$ is given for any $p \in \mathbb{R}$ by

$$
\mathcal{A}_{i j}(x, p)=f_{I}\left(a_{i j}+a_{i k} \frac{\partial \chi^{j}}{\partial y_{k}}\right)(x, y, p) d y,
$$

where $\left\{\chi^{k}\right\}_{k=1}^{d}$ is defined for any $p \in \mathbb{R}$ by

$$
-\frac{\partial}{\partial y_{i}}\left(a_{i k} \frac{\partial \chi^{j}}{\partial y_{k}}\right)(x, y, p)=\frac{\partial}{\partial y_{i}} a_{i j}(x, y, p),
$$


with the periodic boundary condition in $y$ and $\int_{I} \chi^{k}(x, y, p) d y=0$. It is clear that $\mathcal{A}(x, p)$ is also smooth in $x$ and $p$ and satisfies [6, Proposition 3.5]

$$
\lambda I \leq \mathcal{A} \leq \frac{\Lambda^{2}}{\lambda} I
$$

Using Lemma 1.9 and Remark 1.10, for the solution of (5.3), we have

$$
\left\|\nabla V_{\ell}\right\|_{0, I_{\delta}} \leq\left\|\nabla v_{\ell}^{\varepsilon}\right\|_{0, I_{\delta}} \leq \sqrt{\Lambda / \lambda}\left\|\nabla V_{\ell}\right\|_{0, I_{\delta}} .
$$

This gives a bound for $\mathcal{A}_{H}$ :

$$
\lambda I \leq \mathcal{A}_{H} \leq \frac{\Lambda^{2}}{\lambda} I,
$$

which together with (5.23) implies

$$
e(\mathrm{HMM}) \leq 2 \Lambda^{2} / \lambda
$$

This shows that $e(\mathrm{HMM})$ is uniformly bounded.

To simplify the presentation, we will show how to estimate $e(\mathrm{HMM})$ when (5.3) is changed slightly to

$$
\left\{\begin{aligned}
-\operatorname{div}\left(a^{\varepsilon}\left(x, V\left(x_{\ell}\right)\right) \nabla v_{\ell}^{\varepsilon}(x)\right) & =0 & & x \in I_{\delta}\left(x_{\ell}\right), \\
v_{\ell}^{\varepsilon}(x) & =V_{\ell}(x) & & x \in \partial I_{\delta}\left(x_{\ell}\right)
\end{aligned}\right.
$$

and $A_{H}(V, W)$ is changed to

$$
A_{H}(V, W)=\sum_{K \in \mathcal{T}_{H}}|K| \sum_{x_{\ell} \in K} \omega_{\ell} f_{I_{\delta}\left(x_{\ell}\right)} \nabla w_{\ell}^{\varepsilon}(x) \cdot a^{\varepsilon}\left(x, V\left(x_{\ell}\right)\right) \nabla v_{\ell}^{\varepsilon}(x) d x .
$$

If $\delta=\varepsilon$, we replace the Dirichlet boundary condition in (5.26) by the periodic boundary condition, i.e., $v_{\ell}^{\varepsilon}(x)-V_{\ell}(x)$ is periodic on $\partial I_{\varepsilon}\left(x_{\ell}\right)$.

Theorem 5.5. If $(\sqrt{\varepsilon / \delta}+\delta)|\ln H|$ is sufficiently small, then (5.10) and (5.13) hold and

$$
e(\mathrm{HMM}) \leq C\left(\left(\frac{\varepsilon}{\delta}\right)^{1 / 2}+\delta\right) .
$$

In the case of $\delta=\varepsilon$, if $\varepsilon|\ln H|$ is sufficiently small, then (5.10) and (5.13) hold and

$$
e(\mathrm{HMM}) \leq C \varepsilon
$$

In what follows, we concentrate on the first case. The second case when $\delta=\varepsilon$ will be commented on.

Let us first fix more notation. Denote by $\hat{v}_{\ell}^{\varepsilon}$ the solutions of (15.26) with the coefficient $a\left(x, x / \varepsilon, V\left(x_{\ell}\right)\right)$ replaced by $a\left(x_{\ell}, x / \varepsilon, V\left(x_{\ell}\right)\right)$. Similarly we define $w_{\ell}^{\varepsilon}$ to be the solution of (5.26) with $V$ replaced by $W \in X_{H}$. Also, $\hat{w}_{\ell}^{\varepsilon}$ can be defined in the same way and $w_{\ell}^{\varepsilon}$ and $\hat{w}_{\ell}^{\varepsilon}$ can be viewed as the perturbations of $v_{\ell}^{\varepsilon}$ and $\hat{v}_{\ell}^{\varepsilon}$, respectively. Moreover, we define

$$
\begin{aligned}
& a_{V}\left(x_{\ell}\right)=a\left(x_{\ell}, \frac{x}{\varepsilon}, V\left(x_{\ell}\right)\right), \quad a_{W}\left(x_{\ell}\right)=a\left(x_{\ell}, \frac{x}{\varepsilon}, W\left(x_{\ell}\right)\right), \\
& a_{V}(x)=a\left(x, \frac{x}{\varepsilon}, V\left(x_{\ell}\right)\right), \quad a_{W}(x)=a\left(x, \frac{x}{\varepsilon}, W\left(x_{\ell}\right)\right) .
\end{aligned}
$$

Observe that $\hat{v}_{\ell}^{\varepsilon}$ and $\hat{w}_{\ell}^{\varepsilon}$ also satisfy (5.24), and using Lemma 1.8 we have

$$
\left\|\nabla\left(v_{\ell}^{\varepsilon}-\hat{v}_{\ell}^{\varepsilon}\right)\right\|_{0, I_{\delta}} \leq C \delta\left\|\nabla V_{\ell}\right\|_{0, I_{\delta}}, \quad\left\|\nabla\left(w_{\ell}^{\varepsilon}-\hat{w}_{\ell}^{\varepsilon}\right)\right\|_{0, I_{\delta}} \leq C \delta\left\|\nabla W_{\ell}\right\|_{0, I_{\delta}} .
$$


Lemma 5.6. We have

$$
\begin{aligned}
\left\|\nabla\left(\hat{v}_{\ell}^{\varepsilon}-\hat{w}_{\ell}^{\varepsilon}\right)\right\|_{0, I_{\delta}} \leq & C\left(\left|(V-W)\left(x_{\ell}\right)\right|\left(\left\|\nabla V_{\ell}\right\|_{0, I_{\delta}}+\left\|\nabla W_{\ell}\right\|_{0, I_{\delta}}\right)\right. \\
& \left.+\left\|\nabla\left(V_{\ell}-W_{\ell}\right)\right\|_{0, I_{\delta}}\right) .
\end{aligned}
$$

Proof. Observe that

$$
\begin{aligned}
-\operatorname{div}\left(a_{V}\left(x_{\ell}\right) \nabla\left(\hat{v}_{\ell}^{\varepsilon}-V_{\ell}\right)\right) & =\operatorname{div}\left(a_{V}\left(x_{\ell}\right) \nabla V_{\ell}\right), \\
-\operatorname{div}\left(a_{W}\left(x_{\ell}\right) \nabla\left(\hat{w}_{\ell}^{\varepsilon}-W_{\ell}\right)\right) & =\operatorname{div}\left(a_{W}\left(x_{\ell}\right) \nabla W_{\ell}\right) .
\end{aligned}
$$

Both $\hat{v}_{\ell}^{\varepsilon}-V_{\ell}$ and $\hat{w}_{\ell}^{\varepsilon}-W_{\ell}$ vanish on $\partial I_{\delta}\left(x_{\ell}\right)$. Using Lemma 1.8, we obtain

$$
\begin{aligned}
\lambda \| \nabla\left(\hat{v}_{\ell}^{\varepsilon}-V_{\ell}-\right. & \left.\hat{w}_{\ell}^{\varepsilon}+W_{\ell}\right)\left\|_{0, I_{\delta}} \leq \Lambda\right\| \nabla\left(V_{\ell}-W_{\ell}\right) \|_{0, I_{\delta}} \\
& \quad+\max _{x \in I_{\delta}}\left|\left(a_{V}-a_{W}\right)\left(x_{\ell}\right)\right|\left(\left\|\nabla V_{\ell}\right\|_{0, I_{\delta}}+\left\|\nabla\left(\hat{w}_{\ell}^{\varepsilon}-W_{\ell}\right)\right\|_{0, I_{\delta}}\right) .
\end{aligned}
$$

Using (5.24) for $\hat{w}_{\ell}^{\varepsilon}$, we obtain

$$
\left\|\nabla\left(\hat{w}_{\ell}^{\varepsilon}-W_{\ell}\right)\right\|_{0, I_{\delta}} \leq\left\|\nabla \hat{w}_{\ell}^{\varepsilon}\right\|_{0, I_{\delta}}+\left\|\nabla W_{\ell}\right\|_{0, I_{\delta}} \leq C(1+\sqrt{\Lambda / \lambda})\left\|\nabla W_{\ell}\right\|_{0, I_{\delta}},
$$

which together with $\max _{x \in I_{\delta}}\left|\left(a_{V}-a_{W}\right)\left(x_{\ell}\right)\right| \leq C\left|(V-W)\left(x_{\ell}\right)\right|$ gives (5.30).

Next we establish the estimate for $\left(v_{\ell}^{\varepsilon}-\hat{v}_{\ell}^{\varepsilon}\right)-\left(w_{\ell}^{\varepsilon}-\hat{w}_{\ell}^{\varepsilon}\right)$. Let $\psi_{\ell}^{\varepsilon}:=v_{\ell}^{\varepsilon}-\hat{v}_{\ell}^{\varepsilon}$ and $\tilde{\psi}_{\ell}^{\varepsilon}:=w_{\ell}^{\varepsilon}-\hat{w}_{\ell}^{\varepsilon}$. Clearly, $\psi_{\ell}^{\varepsilon}, \tilde{\psi}_{\ell}^{\varepsilon}$ vanish on $\partial I_{\delta}\left(x_{\ell}\right)$ and satisfy

$$
\begin{aligned}
-\operatorname{div}\left(a_{V}(x) \nabla \psi_{\ell}^{\varepsilon}\right) & =\operatorname{div}\left(\left(a_{V}(x)-a_{V}\left(x_{\ell}\right)\right) \nabla \hat{v}_{\ell}^{\varepsilon}\right) \quad x \in I_{\delta}\left(x_{\ell}\right), \\
-\operatorname{div}\left(a_{W}(x) \nabla \tilde{\psi}_{\ell}^{\varepsilon}\right) & =\operatorname{div}\left(\left(a_{W}(x)-a_{W}\left(x_{\ell}\right)\right) \nabla \hat{w}_{\ell}^{\varepsilon}\right) \quad x \in I_{\delta}\left(x_{\ell}\right) .
\end{aligned}
$$

Lemma 5.7. We have

$$
\begin{aligned}
\left\|\nabla\left(\psi_{\ell}^{\varepsilon}-\tilde{\psi}_{\ell}^{\varepsilon}\right)\right\|_{0, I_{\delta}} \leq & C \delta\left(\left|(V-W)\left(x_{\ell}\right)\right|\left(\left\|\nabla V_{\ell}\right\|_{0, I_{\delta}}+\left\|\nabla W_{\ell}\right\|_{0, I_{\delta}}\right)\right. \\
& \left.+\left\|\nabla\left(V_{\ell}-W_{\ell}\right)\right\|_{0, I_{\delta}}\right) .
\end{aligned}
$$

Proof. Using Lemma 1.8, we have

$$
\begin{aligned}
\lambda\left\|\nabla\left(\psi_{\ell}^{\varepsilon}-\tilde{\psi}_{\ell}^{\varepsilon}\right)\right\|_{0, I_{\delta}} \leq & \max _{x \in I_{\delta}}\left|a_{V}(x)-a_{V}\left(x_{\ell}\right)-a_{W}(x)+a_{W}\left(x_{\ell}\right)\right|\left\|\nabla \hat{v}_{\ell}^{\varepsilon}\right\|_{0, I_{\delta}} \\
& +\max _{x \in I_{\delta}}\left|a_{V}(x)-a_{W}(x)\right|\left\|\nabla \tilde{\psi}_{\ell}^{\varepsilon}\right\|_{0, I_{\delta}} \\
& +\max _{x \in I_{\delta}}\left|a_{W}(x)-a_{W}\left(x_{\ell}\right)\right|\left\|\nabla\left(\hat{v}_{\ell}^{\varepsilon}-\hat{w}_{\ell}^{\varepsilon}\right)\right\|_{0, I_{\delta}} .
\end{aligned}
$$

Using

$$
\max _{x \in I_{\delta}}\left|a_{V}(x)-a_{V}\left(x_{\ell}\right)-a_{W}(x)+a_{W}\left(x_{\ell}\right)\right| \leq C \delta\left|(V-W)\left(x_{\ell}\right)\right|,
$$

from $(\underline{5.29})_{2}$, we have $\left\|\nabla \tilde{\psi}_{\ell}^{\varepsilon}\right\|_{0, I_{\delta}} \leq C \delta\left\|\nabla W_{\ell}\right\|_{0, I_{\delta}}$. Collecting the above estimates and using (5.30), we obtain (5.31).

Define

$$
\begin{aligned}
\hat{V}_{\ell}(x) & =V_{\ell}(x)+\varepsilon \chi_{\ell}^{k}\left(x_{\ell}, \frac{x}{\varepsilon}, V\left(x_{\ell}\right)\right) \frac{\partial V_{\ell}}{\partial x_{k}}(x), \\
\hat{W}_{\ell}(x) & =W_{\ell}(x)+\varepsilon \chi_{\ell}^{k}\left(x_{\ell}, \frac{x}{\varepsilon}, W\left(x_{\ell}\right)\right) \frac{\partial W_{\ell}}{\partial x_{k}}(x),
\end{aligned}
$$

where $\left\{\chi_{\ell}^{k}\right\}_{k=1}^{d}$ are the solutions of (5.22) with coefficient replaced by $a_{i j}\left(x_{\ell}, y, p\right)$. 
Denote $\theta_{\ell}^{\varepsilon}=\hat{v}_{\ell}^{\varepsilon}-\hat{V}_{\ell}$ and $\tilde{\theta}_{\ell}^{\varepsilon}=\hat{w}_{\ell}^{\varepsilon}-\hat{W}_{\ell}$. Observe that

$$
\left\{\begin{aligned}
-\operatorname{div}\left(a_{V}\left(x_{\ell}\right) \nabla \theta_{\ell}^{\varepsilon}(x)\right) & =0 & & x \in I_{\delta}\left(x_{\ell}\right), \\
\theta_{\ell}^{\varepsilon}(x) & =-\varepsilon \chi_{\ell}^{k}\left(x_{\ell}, \frac{x}{\varepsilon}, V\left(x_{\ell}\right)\right) \frac{\partial V_{\ell}}{\partial x_{k}} & & x \in \partial I_{\delta}\left(x_{\ell}\right),
\end{aligned}\right.
$$

and

$$
\left\{\begin{aligned}
-\operatorname{div}\left(a_{W}\left(x_{\ell}\right) \nabla \tilde{\theta}_{\ell}^{\varepsilon}(x)\right) & =0 & & x \in I_{\delta}\left(x_{\ell}\right), \\
\tilde{\theta}_{\ell}^{\varepsilon}(x) & =-\varepsilon \chi_{\ell}^{k}\left(x_{\ell}, \frac{x}{\varepsilon}, W\left(x_{\ell}\right)\right) \frac{\partial W_{\ell}}{\partial x_{k}} & & x \in \partial I_{\delta}\left(x_{\ell}\right) .
\end{aligned}\right.
$$

Similarly to (3.9), we have

$$
\left\|\nabla \theta_{\ell}^{\varepsilon}\right\|_{0, I_{\delta}} \leq C\left(\frac{\varepsilon}{\delta}\right)^{1 / 2}\left\|\nabla V_{\ell}\right\|_{0, I_{\delta}}, \quad\left\|\nabla \tilde{\theta}_{\ell}^{\varepsilon}\right\|_{0, I_{\delta}} \leq C\left(\frac{\varepsilon}{\delta}\right)^{1 / 2}\left\|\nabla W_{\ell}\right\|_{0, I_{\delta}} .
$$

Let $\rho^{\varepsilon}$ be defined as in (3.14) and define

$$
\begin{aligned}
& \varphi_{\ell}^{\varepsilon}=\theta_{\ell}^{\varepsilon}+\varepsilon \chi_{\ell}^{k}\left(x_{\ell}, \frac{x}{\varepsilon}, V\left(x_{\ell}\right)\right) \frac{\partial V_{\ell}}{\partial x_{k}}\left(1-\rho^{\varepsilon}\right), \\
& \tilde{\varphi}_{\ell}^{\varepsilon}=\tilde{\theta}_{\ell}^{\varepsilon}+\varepsilon \chi_{\ell}^{k}\left(x_{\ell}, \frac{x}{\varepsilon}, W\left(x_{\ell}\right)\right) \frac{\partial W_{\ell}}{\partial x_{k}}\left(1-\rho^{\varepsilon}\right) .
\end{aligned}
$$

Observe that $\varphi_{\ell}^{\varepsilon}$ and $\tilde{\varphi}_{\ell}^{\varepsilon}$ vanish on $\partial I_{\delta}\left(x_{\ell}\right)$ and satisfy

$$
\begin{aligned}
-\operatorname{div}\left(a_{V}\left(x_{\ell}\right) \nabla \varphi_{\ell}^{\varepsilon}(x)\right)=\operatorname{div}\left(a_{V}\left(x_{\ell}\right) \nabla\left(\varphi_{\ell}^{\varepsilon}-\theta_{\ell}^{\varepsilon}\right)(x)\right) & x \in I_{\delta}\left(x_{\ell}\right), \\
-\operatorname{div}\left(a_{W}\left(x_{\ell}\right) \nabla \tilde{\varphi}_{\ell}^{\varepsilon}(x)\right)=\operatorname{div}\left(a_{W}\left(x_{\ell}\right) \nabla\left(\tilde{\varphi}_{\ell}^{\varepsilon}-\tilde{\theta}_{\ell}^{\varepsilon}\right)(x)\right) & x \in I_{\delta}\left(x_{\ell}\right) .
\end{aligned}
$$

Lemma 5.8. We have

$$
\begin{aligned}
\left\|\nabla\left(\theta_{\ell}^{\varepsilon}-\tilde{\theta}_{\ell}^{\varepsilon}\right)\right\|_{0, I_{\delta}} & \leq C\left(\frac{\varepsilon}{\delta}\right)^{1 / 2}\left(\left\|\nabla\left(V_{\ell}-W_{\ell}\right)\right\|_{0, I_{\delta}}\right. \\
& \left.+\left|(V-W)\left(x_{\ell}\right)\right|\left(\left\|\nabla V_{\ell}\right\|_{0, I_{\delta}}+\left\|\nabla W_{\ell}\right\|_{0, I_{\delta}}\right)\right) .
\end{aligned}
$$

Proof. Using Lemma 1.8, we obtain

$$
\begin{aligned}
\lambda\left\|\nabla\left(\varphi_{\ell}^{\varepsilon}-\tilde{\varphi}_{\ell}^{\varepsilon}\right)\right\|_{0, I_{\delta}} \leq & \max _{x \in I_{\delta}}\left|a_{V}\left(x_{\ell}\right)-a_{W}\left(x_{\ell}\right)\right|\left(\left\|\nabla\left(\varphi_{\ell}^{\varepsilon}-\theta_{\ell}^{\varepsilon}\right)\right\|_{0, I_{\delta}}+\left\|\nabla \tilde{\varphi}_{\ell}^{\varepsilon}\right\|_{0, I_{\delta}}\right) \\
& +\max _{x \in I_{\delta}}\left|a_{W}\left(x_{\ell}\right)\right|\left\|\nabla\left(\varphi_{\ell}^{\varepsilon}-\theta_{\ell}^{\varepsilon}-\tilde{\varphi}_{\ell}^{\varepsilon}+\tilde{\theta}_{\ell}^{\varepsilon}\right)\right\|_{0, I_{\delta} .}
\end{aligned}
$$

A direct computation gives that

$$
\left\|\nabla\left(\varphi_{\ell}^{\varepsilon}-\theta_{\ell}^{\varepsilon}\right)\right\|_{0, I_{\delta}} \leq C\left(\frac{\varepsilon}{\delta}\right)^{\frac{1}{2}}\left\|\nabla V_{\ell}\right\|_{0, I_{\delta}} \quad \text { and } \quad\left\|\nabla\left(\tilde{\varphi}_{\ell}^{\varepsilon}-\tilde{\theta}_{\ell}^{\varepsilon}\right)\right\|_{0, I_{\delta}} \leq C\left(\frac{\varepsilon}{\delta}\right)^{\frac{1}{2}}\left\|\nabla W_{\ell}\right\|_{0, I_{\delta}},
$$

which together with (5.33) gives

$$
\left\|\nabla \tilde{\varphi}_{\ell}^{\varepsilon}\right\|_{0, I_{\delta}} \leq\left\|\nabla \tilde{\theta}_{\ell}^{\varepsilon}\right\|_{0, I_{\delta}}+\left\|\nabla\left(\tilde{\varphi}_{\ell}^{\varepsilon}-\tilde{\theta}_{\ell}^{\varepsilon}\right)\right\|_{0, I_{\delta}} \leq C\left(\frac{\varepsilon}{\delta}\right)^{1 / 2}\left\|\nabla W_{\ell}\right\|_{0, I_{\delta}} .
$$


Note that

$$
\begin{aligned}
\varphi_{\ell}^{\varepsilon}-\theta_{\ell}^{\varepsilon}-\tilde{\varphi}_{\ell}^{\varepsilon}+\tilde{\theta}_{\ell}^{\varepsilon}= & \varepsilon\left(1-\rho^{\varepsilon}\right)\left(\chi_{\ell}^{k}\left(x_{\ell}, \frac{x}{\varepsilon}, V\left(x_{\ell}\right)\right)-\chi_{\ell}^{k}\left(x_{\ell}, \frac{x}{\varepsilon}, W\left(x_{\ell}\right)\right)\right) \frac{\partial V_{\ell}}{\partial x_{k}} \\
& +\varepsilon\left(1-\rho^{\varepsilon}\right) \chi_{\ell}^{k}\left(x_{\ell}, \frac{x}{\varepsilon}, W\left(x_{\ell}\right)\right) \frac{\partial}{\partial x_{k}}\left(V_{\ell}-W_{\ell}\right) .
\end{aligned}
$$

Using the continuity of $\left\{\chi_{\ell}^{k}\right\}_{k=1}^{d}$, a direct computation gives

$\left\|\nabla\left(\varphi_{\ell}^{\varepsilon}-\theta_{\ell}^{\varepsilon}-\tilde{\varphi}_{\ell}^{\varepsilon}+\tilde{\theta}_{\ell}^{\varepsilon}\right)\right\|_{0, I_{\delta}} \leq C\left(\frac{\varepsilon}{\delta}\right)^{1 / 2}\left(\left|(V-W)\left(x_{\ell}\right)\right|\left\|\nabla V_{\ell}\right\|_{0, I_{\delta}}+\left\|\nabla\left(V_{\ell}-W_{\ell}\right)\right\|_{0, I_{\delta}}\right)$.

Adding these up, we obtain (5.34).

In the next lemma, we shall prove that $E(V, W)$ has certain continuity with respect to $V$.

Lemma 5.9. For any $V, W, Z \in X_{H}$ satisfying $\|V\|_{1, \infty}+\|W\|_{1, \infty} \leq M$, there exists a constant $C(M)$ such that (5.13) holds with $\eta(M)=C(M)(\sqrt{\varepsilon / \delta}+\delta)$.

Proof. Using the definition of $v_{\ell}^{\varepsilon}$ and noticing that $v_{\ell}^{\varepsilon}=\left(v_{\ell}^{\varepsilon}-\hat{v}_{\ell}^{\varepsilon}\right)+\theta_{\ell}^{\varepsilon}+\hat{V}_{\ell}$, we obtain

$$
\begin{aligned}
& f_{I_{\delta}} \nabla z_{\ell}^{\varepsilon} \cdot a_{V}(x) \nabla v_{\ell}^{\varepsilon} d x=f_{I_{\delta}} \nabla Z_{\ell} \cdot a_{V}(x) \nabla v_{\ell}^{\varepsilon} d x \\
& =\nabla Z_{\ell} \cdot\left(f_{I_{\delta}} a_{V}(x)\left(\nabla\left(v_{\ell}-\hat{v}_{\ell}^{\varepsilon}\right)+\nabla \theta_{\ell}^{\varepsilon}+\nabla \hat{V}_{\ell}\right) d x\right),
\end{aligned}
$$

which together with $f_{I_{\kappa \varepsilon}} \nabla Z_{\ell} \cdot a_{V}\left(x_{\ell}\right) \nabla \hat{V}_{\ell} d x=\nabla Z\left(x_{\ell}\right) \cdot \mathcal{A}\left(x_{\ell}, V\left(x_{\ell}\right)\right) \nabla V\left(x_{\ell}\right)$ gives

$$
E(V, Z)-E(W, Z)=: I_{1}+I_{2}+I_{3},
$$

with

$$
\begin{aligned}
& I_{1}=\nabla Z_{\ell} \cdot\left[f_{I_{\delta}}\left(a_{V}(x) \nabla\left(v_{\ell}^{\varepsilon}-\hat{v}_{\ell}^{\varepsilon}\right)-a_{W}(x) \nabla\left(w_{\ell}^{\varepsilon}-\hat{w}_{\ell}^{\varepsilon}\right)\right) d x\right], \\
& I_{2}=\nabla Z_{\ell} \cdot\left[f_{I_{\delta}} a_{V}(x) \nabla \theta_{\ell}^{\varepsilon}-a_{W}(x) \nabla \tilde{\theta}_{\ell}^{\varepsilon} d x\right],
\end{aligned}
$$

and

$$
\begin{aligned}
I_{3}=\nabla Z_{\ell} \cdot\left[f_{I_{\delta}} a_{V}(x) \nabla \hat{V}_{\ell} d x-f_{I_{\kappa \varepsilon}} a_{V}\left(x_{\ell}\right) \nabla \hat{V}_{\ell} d x\right. \\
\left.\quad-f_{I_{\delta}} a_{W}(x) \nabla \hat{W}_{\ell} d x+f_{I_{\kappa \varepsilon}} a_{W}\left(x_{\ell}\right) \nabla \hat{W}_{\ell} d x\right] .
\end{aligned}
$$

$I_{1}$ can be decomposed into

$$
I_{1}=\nabla Z_{\ell} \cdot\left[f_{I_{\delta}}\left(a_{V}(x)-a_{W}(x)\right) \nabla\left(v_{\ell}^{\varepsilon}-\hat{v}_{\ell}^{\varepsilon}\right) d x+f_{I_{\delta}} a_{W}(x) \nabla\left(\psi_{\ell}^{\varepsilon}-\tilde{\psi}_{\ell}^{\varepsilon}\right) d x\right] .
$$

Using (5.29) and (5.31), we bound $I_{1}$ as

$$
\left|I_{1}\right| \leq C \delta\left(\left|\nabla\left(V_{\ell}-W_{\ell}\right)\right|+\left|(V-W)\left(x_{\ell}\right)\right|\left(\left|\nabla V_{\ell}\right|+\left|\nabla W_{\ell}\right|\right)\right)\left|\nabla Z_{\ell}\right| .
$$

Similarly, using Lemma 5.8, we bound $I_{2}$ as

$$
\left|I_{2}\right| \leq C\left(\frac{\varepsilon}{\delta}\right)^{1 / 2}\left(\left|\nabla\left(V_{\ell}-W_{\ell}\right)\right|+\left|(V-W)\left(x_{\ell}\right)\right|\left(\left|\nabla V_{\ell}\right|+\left|\nabla W_{\ell}\right|\right)\right)\left|\nabla Z_{\ell}\right| .
$$


$I_{3}$ can be rewritten as

$$
\begin{aligned}
I_{3}= & \nabla Z_{\ell} \cdot f_{I_{\delta}}\left(\left(a_{V}(x)-a_{V}\left(x_{\ell}\right)\right) \nabla \hat{V}_{\ell}-\left(a_{W}(x)-a_{W}\left(x_{\ell}\right)\right) \nabla \hat{W}_{\ell}\right) d x \\
& -\nabla Z_{\ell} \cdot\left(\frac{1}{|\kappa \varepsilon|^{d}}-\frac{1}{\delta^{d}}\right) \int_{I_{\delta}}\left(a_{V}\left(x_{\ell}\right) \nabla \hat{V}_{\ell}-a_{W}\left(x_{\ell}\right) \nabla \hat{W}_{\ell}\right) d x \\
+ & \frac{1}{|\kappa \varepsilon|^{d}} \nabla Z_{\ell} \cdot \int_{I_{\delta} \backslash I_{\kappa \varepsilon}}\left(a_{V}\left(x_{\ell}\right) \nabla \hat{V}_{\ell}-a_{W}\left(x_{\ell}\right) \nabla \hat{W}_{\ell}\right) d x .
\end{aligned}
$$

As in $I_{1}$ and using (5.32), we bound $I_{3}$ as

$$
\left|I_{3}\right| \leq C\left(\frac{\varepsilon}{\delta}+\delta\right)\left(\left|\nabla\left(V_{\ell}-W_{\ell}\right)\right|+\left|(V-W)\left(x_{\ell}\right)\right|\left(\left|\nabla V_{\ell}\right|+\left|\nabla W_{\ell}\right|\right)\right)\left|\nabla Z_{\ell}\right| .
$$

Adding these up, we get

$$
\begin{aligned}
|E(V, Z)-E(W, Z)| \leq & C\left(\frac{\varepsilon}{\delta}+\delta+\left(\frac{\varepsilon}{\delta}\right)^{1 / 2}\right)\left(\left|\nabla\left(V_{\ell}-W_{\ell}\right)\right|\right. \\
& \left.+\left|(V-W)\left(x_{\ell}\right)\right|\left(\left|\nabla V_{\ell}\right|+\left|\nabla W_{\ell}\right|\right)\right)\left|\nabla Z_{\ell}\right| .
\end{aligned}
$$

Consequently we obtain

$$
\begin{aligned}
|K| \sum_{x_{\ell} \in K} \omega_{\ell}|E(V, Z)-E(W, Z)| \leq C\left(\delta+\left(\frac{\varepsilon}{\delta}\right)^{1 / 2}\right)\|\nabla(V-W)\|_{0, K}\|\nabla Z\|_{0, K} \\
+C\left(\delta+\left(\frac{\varepsilon}{\delta}\right)^{1 / 2}\right)\|V-W\|_{L^{\infty}(K)}\left(\|\nabla V\|_{0, K}+\|\nabla W\|_{0, K}\right)\|\nabla Z\|_{0, K} .
\end{aligned}
$$

Using the inverse inequality (1.21) on each element, we obtain

$$
\begin{aligned}
\|V-W\|_{L^{\infty}(K)}\|\nabla V\|_{0, K} & \leq C H_{K}^{-d / 2}\|V-W\|_{0, K} H_{K}^{d / 2}\|\nabla V\|_{L^{\infty}(K)} \\
& =C\|V-W\|_{0, K}\|\nabla V\|_{L^{\infty}(K)} .
\end{aligned}
$$

Similarly $\|V-W\|_{L^{\infty}(K)}\|\nabla W\|_{0, K} \leq C\|V-W\|_{0, K}\|\nabla W\|_{L^{\infty}(K)}$. A combination of the above estimates gives (5.13) with $\eta(M)=C(M)(\sqrt{\varepsilon / \delta}+\delta)$.

Proof of the first case in Theorem5.5 Let $W=0$ in (5.35) and note that $V \in$ $W^{1, \infty}(D)$. We obtain (5.27). Therefore if $(\sqrt{\varepsilon / \delta}+\delta)|\ln H|$ is sufficiently small, then $e(\mathrm{HMM})^{1 / 2}|\ln H|$ can be smaller than any given threshold; this verifies (5.10).

Next let $\eta(M)=C(M)(\sqrt{\varepsilon / \delta}+\delta)$. Then if $(\sqrt{\varepsilon / \delta}+\delta)|\ln H|$ is sufficiently small, we have $\eta(M)<1$; this verifies (5.13).

Remark 5.10. Compared to the linear case, the upper bound for $e(\mathrm{HMM})$ for the case when $\delta / \varepsilon \notin \mathbb{Z}$ degrades to $\sqrt{\varepsilon / \delta}$. This is due to the fact that $A_{H}$ is nonsymmetric.

In the case of $\delta=\varepsilon$, note that $\hat{v}_{\ell}^{\varepsilon}=\hat{V}_{\ell}$ and $\hat{w}_{\ell}^{\varepsilon}=\hat{W}_{\ell}$. So a direct calculation gives Lemma 5.6 for this case. Lemma 5.7 is also valid with $\delta$ replaced by $\varepsilon$. We also have $\theta_{\ell}^{\varepsilon}=0$ and $\tilde{\theta}_{\ell}^{\varepsilon}=0$. Observing that for any $V, Z \in X_{H}$,

$$
f_{I_{\varepsilon}\left(x_{\ell}\right)} \nabla z_{\ell}^{\varepsilon} \cdot a_{V}\left(x_{\ell}\right) \nabla \hat{v}_{\ell}^{\varepsilon} d x=f_{K} \nabla Z_{\ell} \cdot \mathcal{A}\left(x_{\ell}, V\left(x_{\ell}\right)\right) \nabla V_{\ell} d x
$$


we may rewrite $E(V, Z)$ as

$$
E(V, Z)=f_{I_{\varepsilon}} \nabla z_{\ell}^{\varepsilon} \cdot a_{V}(x) \nabla\left(v_{\ell}^{\varepsilon}-\hat{v}_{\ell}^{\varepsilon}\right) d x+f_{I_{\varepsilon}} \nabla z_{\ell}^{\varepsilon} \cdot\left(a_{V}(x)-a_{V}\left(x_{\ell}\right)\right) \nabla \hat{v}_{\ell}^{\varepsilon} d x .
$$

Consequently, (5.13) holds with $\eta(M)=C(M) \varepsilon$.

Proof of the second case in Theorem 5.5. Let $W=0$ in (5.35) and note that $V$ $\in W^{1, \infty}(D)$. We obtain (5.28). Therefore if $\varepsilon|\ln H|$ is sufficiently small, then $e(\mathrm{HMM})^{1 / 2}|\ln H|$ can be smaller than any given threshold; this verifies (5.10).

Next let $\eta(M)=C(M) \varepsilon$. Then if $\varepsilon|\ln H|$ is sufficiently small, we have $\eta(M)<1$. This proves (5.13).

\section{Appendix A. Estimating e(HMM) For Problems WITH RANDOM COEFFICIENTS}

Here we estimate $e(\mathrm{HMM})$ for the random case. Our basic strategy follows that of [43].

Denote a probability space by $(\Omega, \mathcal{F}, P)$ and let $a(y, \omega)=\left(a_{i j}(y, \omega)\right)$ be a random field whose statistics are invariant under integer shifts and which satisfies the uniform ellipticity condition that there exist constants $\lambda$ and $\Lambda$ such that

$$
\lambda|\xi|^{2} \leq a_{i j}(y, \omega) \xi_{i} \xi_{j} \leq \Lambda|\xi|^{2},
$$

for almost all $y \in \mathbb{R}^{d}$ and $\omega \in \Omega$. For $j=1, \cdots, d$, denote by $\varphi_{j}(y, \omega)$ the solutions of the cell problem:

$$
\mathcal{L}_{y} \varphi_{j}:=-\operatorname{div}_{y}\left(a_{i j}(y, \omega) \nabla_{y} \varphi_{j}\right)=\operatorname{div}_{y}\left(a_{i j} \cdot e_{j}\right),
$$

where $\left\{e_{j}\right\}_{j=1}^{d}$ are the standard basis in $\mathbb{R}^{d} . \nabla \varphi_{j}$ is required to be stationary under integer shift. The existence of $\varphi_{j}$ is proved in [28, 36]. In general $\varphi_{j}$ is not stationary. The homogenized coefficient $\mathcal{A}$ [28, 36] is given by

$$
\mathcal{A}=\langle a(I+\nabla \varphi)\rangle \text {. }
$$

Here and in the following, we use the notation

$$
\langle f\rangle=\mathbb{E} \int_{[0,1]^{d}} f(y) d y
$$

and

$$
[f ; m]=\frac{1}{m^{d}} \int_{[0, m]^{d}} f(y) d y,
$$

where $\mathbb{E}$ denotes the expectation in the probability space $(\Omega, \mathcal{F}, P)$.

As in [43], we will consider the following auxiliary problem:

$$
\mathcal{L} u+\rho u=f,
$$

for any $\rho>0$, where $f$ is of the form

$$
f=\sum_{j=1}^{d} D_{j} g_{j}+h,
$$

with $g_{j}, h \in \rho_{G}$ which is defined as

$$
\rho_{G}:=\left\{\psi \mid\left\langle|\psi|^{2}\right\rangle \leq G^{2}\right\}
$$

and $\psi$ is a random field whose statistics are stationary with respect to integer shifts. 
The solution of A.2 can be expressed with the help of a diffusion process $\eta$ generated by the operator $\mathcal{L}$.

For each fixed realization of $\{a(y, \cdot)\}$, denote by $\eta_{x}$ the diffusion process generated by $\mathcal{L}_{1}$ and starting from $x$ at $t=0$, and denote by $M_{x}$ the expectation with respect to $\eta_{x}$. Let

$$
\Gamma(\tau)=\int_{0}^{\tau} f(\eta(t)) e^{-\rho t} d t .
$$

Then it is well known 21] that the solution of (A.2) is given by

$$
u(x)=M_{x} \Gamma(\infty) .
$$

The following results are either standard or proved in [28, 43].

Lemma A.1. If $u$ is the solution of (A.2), then there exists a constant $C$ independent of $\rho$ such that

$$
\begin{aligned}
\left\langle|\nabla u|^{2}\right\rangle+\rho\left\langle u^{2}\right\rangle & \leq C\left(\left\langle g^{2}\right\rangle+\frac{1}{\rho}\left\langle h^{2}\right\rangle\right), \\
\left\langle\left(M_{x} \Gamma(\infty)\right)^{2}\right\rangle^{1 / 2} & \leq C \frac{G^{2}}{\rho}, \\
\left\langle M_{x}(\Gamma(\infty)-\Gamma(s))^{2}\right\rangle & \leq C \frac{G^{2}}{\rho} e^{-2 s \rho} .
\end{aligned}
$$

Because of the lowest order term $\rho u$, the Green's function associated with the operator $\mathcal{L}_{1}+\rho I$ decays exponentially with rate $\mathcal{O}(\sqrt{\rho})$. To make this statement precise, we define the norm $\|x\|:=\max _{i}\left|x_{i}\right|$, and

$$
Q_{\rho}:=\left\{x \in \mathbb{R}^{d} \mid\|x\| \leq\left(\frac{1}{\rho}\right)^{\frac{1}{2}[\ln (1 / \rho)]^{1 / 2}}\right\} .
$$

Let $\tau$ be the first exit time of $Q_{\rho}$ starting at $x \in Q_{\rho}$. Let $\hat{\varphi}_{\rho}(x)=M_{x} \Gamma(\tau)$.

Lemma A.2 (43]). If $\rho$ is sufficiently small, then

$$
\begin{array}{r}
\mathbb{E} \int_{\|x\| \leq 10}\left|\varphi_{\rho}(x)-\hat{\varphi}_{\rho}(x)\right|^{2} d x \leq C G^{2} e^{-C|\ln (1 / \rho)|^{2}}, \\
\mathbb{E} \int_{\|x\| \leq 1}\left|\nabla \varphi_{\rho}(x)-\nabla \hat{\varphi}_{\rho}(x)\right|^{2} d x \leq C G^{2} e^{-C|\ln (1 / \rho)|^{2}} .
\end{array}
$$

To prepare for the discussion on the consequence of the mixing condition, we mention

Lemma A.3 (43]). Let $\left\{a_{i j}, g_{j}\right\}$ and $\left\{\tilde{a}_{i j}, \tilde{g}_{j}\right\}$ be two sets of data such that

$$
\left\{a_{i j}(y), g_{j}(y)\right\}=\left\{\tilde{a}_{i j}(y), \tilde{g}_{j}(y)\right\}
$$

for $y \notin B$, where $B$ is a domain in $\mathbb{R}^{d}$, and let $\varphi_{\rho}$ and $\hat{\varphi}_{\rho}$ be the solutions of (A.2) associated with $\left\{a_{i j}, g_{j}\right\}$ and $\left\{\tilde{a}_{i j}, \tilde{g}_{j}\right\}$, respectively (with $h=0$ ). Then

$$
\int_{\mathbb{R}^{d}}\left|\varphi_{\rho}(x)-\tilde{\varphi}_{\rho}(x)\right|^{2} d x \leq \frac{C}{\rho} \int_{\mathbb{R}^{d}}\left(G^{2}+\left|\nabla \varphi_{\rho}(x)\right|^{2}\right) I_{B}(x) d x,
$$

where $I_{B}$ is the indicator function of the domain $B$. 
Now we introduce the crucial mixing condition 22]. Let $B$ be a domain in $\mathbb{R}^{d}$. Denote by $\mathcal{F}(B)$ the $\sigma$-algebra generated by $\left\{a_{i j}(y, \omega), y \in B\right\}$. Let $\xi, \eta$ be two random variables that are measurable with respect to $\mathcal{F}\left(B_{1}\right)$ and $\mathcal{F}\left(B_{2}\right)$, respectively. Then

$$
\frac{|\mathbb{E} \xi \eta-\mathbb{E} \xi \mathbb{E} \eta|}{\left(\mathbb{E} \xi^{2}\right)^{1 / 2}\left(\mathbb{E} \eta^{2}\right)^{1 / 2}} \leq e^{-\lambda q},
$$

where $q=\operatorname{dist}\left(B_{1}, B_{2}\right), \lambda>0$ is a fixed constant.

Lemma A.4. Under condition (A), we have

$$
\mathbb{E}\left[\varphi_{\rho} ; m\right]^{2} \leq C\left(\frac{G^{2}}{\rho}\left(\frac{|\ln (1 / \rho)|^{2}}{\rho^{1 / 2} m}\right)^{d}+e^{-c(\ln (1 / \rho))^{2}}\right) .
$$

Proof. For $\ell=\left(\ell_{1}, \cdots, \ell_{d}\right) \in \mathbb{Z}^{d} \cap[0, m)^{d}$ : $=\mathbb{Z}_{m}^{d}$, denote by $I^{\ell}$ the cube of size 1 centered at $\ell+\frac{1}{2}=\left(\ell_{1}+\frac{1}{2}, \cdots, \ell_{d}+\frac{1}{2}\right)$, and let $\varphi^{\ell}=\int_{I^{\ell}} \varphi_{\rho}(x) d x$. Then

$$
\left[\varphi_{\rho} ; m\right]=\frac{1}{m^{d}} \sum_{\ell \in \mathbb{Z}_{m}^{d}} \varphi^{\ell}
$$

We first estimate $\mathbb{E}\left(\varphi^{\ell} \varphi^{k}\right)$. If $|\ell-k| \leq C \rho^{-1 / 2}|\ln (1 / \rho)|^{2}$, then

$$
\mathbb{E}\left(\varphi^{\ell} \varphi^{k}\right) \leq\left\langle\varphi_{\rho}^{2}(x)\right\rangle^{1 / 2}\left\langle\varphi_{\rho}^{2}(x)\right\rangle^{1 / 2} \leq C \frac{G^{2}}{\rho} .
$$

If $|\ell-k| \geq C \rho^{-1 / 2}|\ln (1 / \rho)|^{2}$, then let $B_{1}:=\ell+B\left(\rho^{-1 / 2}|\ln (1 / \rho)|^{2}\right)$ and $B_{2}:=$ $k+B\left(\rho^{-1 / 2}|\ln (1 / \rho)|^{2}\right)$, where $B(s)$ is a ball of size $s$ in the norm $\|\cdot\|$. Denote by $\tilde{\varphi}_{1}(x)$ the solution of A.2) in which the coefficient $\left(a_{i j}(y, \omega)\right)$ is modified in $\mathbb{R}^{d} \backslash B_{1}$ such that it is measurable with respect to $\mathcal{F}\left(B_{1}\right)$, and similarly denote by $\tilde{\varphi}_{2}(x)$ the solution of A.2 in which the coefficient $\left(a_{i j}(y, \omega)\right)$ is modified in $\mathbb{R}^{d} \backslash B_{2}$ such that it is measurable with respect to $\mathcal{F}\left(B_{2}\right)$. The modified coefficients $\left(\tilde{a}_{i j}(y, \omega)\right)$ should still satisfy the condition on $a_{i j}$ listed in the beginning of this subsection. From $\tilde{\varphi}_{1}$ and $\tilde{\varphi}_{2}$, we can similarly define $\tilde{\varphi}_{1}^{\ell}$ and $\tilde{\varphi}_{2}^{\ell}$. Using (A.6), we have

$$
\begin{aligned}
\mathbb{E}\left(\tilde{\varphi}_{1}^{\ell}-\tilde{\varphi}^{\ell}\right)^{2} & \leq C G^{2} e^{-C|\ln (1 / \rho)|^{2}}, \\
\mathbb{E}\left(\tilde{\varphi}_{1}^{k}-\tilde{\varphi}^{k}\right)^{2} & \leq C G^{2} e^{-C|\ln (1 / \rho)|^{2}} .
\end{aligned}
$$

Since

$$
\begin{aligned}
\mathbb{E}\left(\varphi^{\ell} \varphi^{k}\right)= & \mathbb{E}\left(\tilde{\varphi}^{\ell} \tilde{\varphi}^{k}\right)+\mathbb{E}\left(\varphi^{\ell}-\tilde{\varphi}_{1}^{\ell}\right) \tilde{\varphi}_{2}^{k}+\mathbb{E} \tilde{\varphi}_{1}^{\ell}\left(\varphi^{k}-\tilde{\varphi}_{2}^{k}\right) \\
& +\mathbb{E}\left(\varphi^{\ell}-\tilde{\varphi}_{1}^{\ell}\right)\left(\varphi^{k}-\tilde{\varphi}_{2}^{k}\right)
\end{aligned}
$$

and

$$
\begin{aligned}
\left|\mathbb{E} \tilde{\varphi}_{1}^{\ell}\right|,\left|\mathbb{E} \tilde{\varphi}_{2}^{k}\right| & \leq C G e^{-C|\ln (1 / \rho)|^{2}}, \\
\left|\mathbb{E} \tilde{\varphi}_{1}^{\ell} \tilde{\varphi}_{2}^{k}\right| & \leq C\left(\frac{G^{2}}{\rho} e^{-C|\ell-k|}+G^{2} e^{-C|\ln (1 / \rho)|^{2}}\right),
\end{aligned}
$$

we thus have

$$
\left|\mathbb{E} \varphi^{\ell} \varphi^{k}\right| \leq C\left(\frac{G^{2}}{\rho} e^{-C|\ell-k|}+G^{2} e^{-C|\ln (1 / \rho)|^{2}}\right)
$$


Hence

$$
\begin{aligned}
\mathbb{E}\left[\varphi_{\rho} ; m\right]^{2}= & \frac{1}{m^{2 d}} \sum_{\ell, k \in \mathbb{Z}_{m}^{d}} \mathbb{E} \varphi^{\ell} \varphi^{k} \\
= & \frac{1}{m^{2 d}}\left(\sum_{|\ell-k| \leq \rho^{-1 / 2}|\ln (1 / \rho)|^{2}} \mathbb{E} \varphi^{\ell} \varphi^{k}+\sum_{|\ell-k|>\rho^{-1 / 2}|\ln (1 / \rho)|^{2}} \mathbb{E} \varphi^{\ell} \varphi^{k}\right) \\
\leq & \frac{1}{m^{2 d}}\left(\frac{G^{2}}{\rho} m^{d}\left(\rho^{-1 / 2}|\ln (1 / \rho)|^{2}\right)^{d}+m^{2 d} G^{2} e^{-C|\ln (1 / \rho)|^{2}}\right. \\
& \left.\times \frac{G^{2}}{\rho} e^{-C|\ell-k|}\right) \\
\leq & C \frac{G^{2}}{\rho}\left(\frac{\ln (1 / \rho)}{\rho^{1 / 2} m}\right)^{d}+e^{-C|\ln (1 / \rho)|^{2}} .
\end{aligned}
$$

Proceeding along the same line as in [43, Theorem 2.1], using condition (A), we have

Lemma A.5. For any $0<\gamma<1 / 2$, under condition (A), there exists a constant $C$ such that

$$
\left|\mathcal{A}-\left\langle a\left(I+\nabla \chi_{\rho}\right)\right\rangle\right| \leq C \rho^{\frac{d-2-2 \gamma}{4+d}}
$$

where $\chi_{\rho}=\left\{\chi_{k, \rho}\right\}_{k=1}^{d}$, and $\chi_{k, \rho}$ is the solution of A.1 with $g=\left(a_{k 1}, \cdots, a_{k d}\right)$.

Now we are ready to estimate $e(\mathrm{HMM})$. Define $m=\frac{\delta}{2 \varepsilon}$ and denote by $\varphi_{j}^{m}$ the solution of A.1. on $I_{m}=[0, m]^{d}$ with the boundary condition that $\varphi_{j}^{m}(y)=0$ on $\partial I_{m}$, and let $\varphi^{m}=\left(\varphi_{1}^{m}, \cdots, \varphi_{d}^{m}\right)$. Then

$$
e(\mathrm{HMM})=\left|\mathcal{A}-\left[a\left(I+\nabla \varphi^{m}\right)\right]_{m}\right| \text {. }
$$

Define $\varphi_{\rho}, \varphi_{\rho}^{m}$ similarly. We have

$$
e(\mathrm{HMM}) \leq E_{1}+E_{2}+E_{3},
$$

where

$$
\begin{aligned}
& E_{1}=\left|\mathcal{A}-\left[a\left(I+\nabla \varphi_{\rho}\right)\right]_{m}\right|, \\
& E_{2}=\left|\left[a\left(I+\nabla \varphi_{\rho}\right)-a\left(I+\nabla \varphi_{\rho}^{m}\right)\right]_{m}\right|, \\
& E_{3}=\left|\left[a \nabla\left(\varphi_{\rho}^{m}-\varphi^{m}\right)\right]_{m}\right| .
\end{aligned}
$$

Obviously,

$$
E_{1}=\left|\mathcal{A}-\left\langle a\left(I+\nabla \varphi_{\rho}\right)\right\rangle+[\tilde{\psi}]_{m}\right|,
$$

with $\tilde{\psi}=\left\langle a\left(I+\nabla \varphi_{\rho}\right)\right\rangle-a\left(I+\nabla \varphi_{\rho}\right)$. It was proved in [43, Lemma 2.5] that

$$
\mathbb{E}\left|[\tilde{\psi}]_{m}\right| \leq\left(\mathbb{E}[\tilde{\psi}]_{m}^{2}\right)^{1 / 2} \leq C\left(\frac{|\ln (1 / \rho)|^{2}}{\rho^{1 / 2} m}\right)^{d / 2} .
$$

The above inequality together with Lemma A.5 gives

$$
\mathbb{E} E_{1} \leq C\left(G \rho^{\frac{d-2-2 \gamma}{4+d}}+\left(\frac{|\ln (1 / \rho)|^{2}}{\rho^{1 / 2} m}\right)^{d / 2}\right) \text {. }
$$


To estimate $E_{2}$, denote by $\tau_{m}$ the first exit time for the domain $I_{2 m}$. Then $\varphi_{\rho}^{2 m}=M_{x} \Gamma\left(\tau_{m}\right)$. For any $s>0$,

$$
\begin{aligned}
\left|\varphi_{\rho}-\varphi_{\rho}^{2 m}\right|= & \left|M_{x}\left(\Gamma(\infty)-\Gamma\left(\tau_{m}\right)\right)\right| \\
\leq & M_{x}\left\{|\Gamma(\infty)|+\left|\Gamma\left(\tau_{m}\right)\right| ; \tau_{m} \leq s\right\} \\
& +M_{x}\left\{e^{-s \rho} M_{\rho(s)}\left|\Gamma(\infty)-\Gamma\left(\tau_{m}\right)\right| ; \tau_{m}>s\right\} \\
\leq & C\left(M_{x}\left(\left(\Gamma(\infty)^{2}+\Gamma\left(\tau_{m}\right)^{2}\right)\right)^{1 / 2}\left\{P_{x}\left(\tau_{m} \leq s\right)^{1 / 2}+e^{-s \rho}\right\} .\right.
\end{aligned}
$$

Since

$$
P_{x}\left\{\tau_{m} \leq s\right\} \leq e^{-C m^{2} / s},
$$

we get

$$
\mathbb{E}\left[\left|\varphi_{\rho}-\varphi_{\rho}^{2 m}\right|^{2}\right]_{2 m} \leq C \frac{G^{2}}{\rho}\left(e^{-C m^{2} / s}+e^{-s \rho}\right)^{2} .
$$

Optimizing in $s$, we get

$$
\mathbb{E}\left[\left|\varphi_{\rho}-\varphi_{\rho}^{2 m}\right|^{2}\right]_{2 m} \leq C \frac{G^{4}}{\rho^{2}} e^{-C m \rho^{1 / 2}} .
$$

Using standard interior estimates, we have

$$
\mathbb{E} E_{2} \leq\left(\mathbb{E}\left[\left|\nabla\left(\varphi_{\rho}-\varphi_{\rho}^{m}\right)\right|^{2}\right]_{m}\right)^{1 / 2} \leq \frac{C}{m}\left(\mathbb{E}\left[\left|\varphi_{\rho}-\varphi_{\rho}^{2 m}\right|^{2}\right]_{2 m}\right)^{1 / 2} \leq \frac{C G^{2}}{m \rho} e^{-C m \rho^{1 / 2}} .
$$

As for $E_{3}$, proceeding along the same line as in the estimate of $E_{1}$, we get

$$
\mathbb{E} E_{3} \leq C\left(G^{2} \rho^{\frac{d-2-2 \gamma}{4+d}}+\left(\frac{|\ln (1 / \rho)|^{2}}{\rho^{1 / 2} m}\right)^{d / 2}\right) .
$$

To sum up, we have

$$
\mathbb{E} e(\mathrm{HMM}) \leq C\left(G^{2} \rho^{\frac{d-2-2 \gamma}{4+d}}+\left(\frac{|\ln (1 / \rho)|^{2}}{\rho^{1 / 2} m}\right)^{d / 2}+\frac{C G^{2}}{m \rho} e^{-C m \rho^{1 / 2}}\right) .
$$

Optimizing in $\rho$ with respect to the first two terms, we get $\rho_{0}=m^{-\frac{2 d}{d+4 \alpha}}$ with $\alpha=(d-2-2 \gamma) /(d+4)$. Hence

$$
\mathbb{E} e(\mathrm{HMM}) \leq C\left(\frac{|\ln m|^{d}}{m^{\kappa}}+\frac{C G^{2}}{m \rho_{0}} e^{-C m \rho_{0}^{1 / 2}}\right) \leq C \frac{|\ln m|^{d}}{m^{\kappa}},
$$

with

$$
\kappa=\frac{d / 2}{1+\frac{d(d+4) / 4}{d-2-2 \gamma}}, \quad 0<\gamma<\frac{1}{2} .
$$

Obviously, the $|\ln m|^{d}$ factor in (A.11) can be absorbed into the factor $m^{-\kappa}$. This proves Theorem 1.3 for $d=3$.

Remark A.6. The estimate (A.11) is unlikely to be optimal. If $d=1$, a direct calculation gives

$$
\mathbb{E} e(\mathrm{HMM}) \leq C\left(\frac{\varepsilon}{\delta}\right)^{1 / 2},
$$


whereas the estimate (A.11) does not apply for $d=1$ and $d=2$. We may use the techniques in [13] to derive improved bounds for $e(\mathrm{HMM})$ if the magnitude of the oscillation in the coefficients $\left(a_{i j}^{\varepsilon}\right)$ is sufficiently small.

\section{REFERENCES}

1. R.A. Adams and J.J. F. Fournier, Sobolev Spaces, second edition, Academic Press, New York, 2003.

2. G. Allaire, Homogenization and two-scale convergence, SIAM J. Math. Anal., 23(1992), 14821518. MR 1185639 (93k:35022)

3. I. Babuska, Homogenization and its applications, mathematical and computational problems, Numerical Solutions of Partial Differential Equations-III (SYNSPADE 1975, College Park, MD, May 1975) (B. Hubbard ed.), Academic Press, New York, 1976, pp. 89-116. MR0502025 $(58: 19215)$

4. I. Babuska, Solution of interface by homogenization. I, II, III, SIAM J. Math. Anal., 7 (1976), 603-634, 635-645, 8 (1977), 923-937. MR0509273 (58:23013a) MR0509277 (58:23013b) MR0509282 (58:23014)

5. A. Bensoussan, J.L. Lions and G.C. Papanicolaou, Asymptotic Analysis for Periodic Structures, North-Holland, Amsterdam, 1978. MR0503330 (82h:35001)

6. L. Boccardo and F. Murat, Homogénéisation de problémes quasi-linéaires, Publ. IRMA, Lille., 3 (1981), no. 7, 13-51.

7. J.F. Bourgat, Numerical experiments of the homogenization method for operators with periodic coefficients, Lecture Notes in Mathematics, Vol. 707. Springer-Verlag, 1977, pp. 330-356. MR0540121 (80e:65108)

8. A. Brandt, Multi-level adaptive solutions to boundary-value problems, Math. Comp., 31 (1977), 333-390. MR0431719 (55:4714)

9. S.C. Brenner and L.R. Scott, The Mathematical Theory of Finite Element Methods, SpringerVerlag, New York, 1994. MF,1278258 (95f:65001)

10. P.G. Ciarlet, The Finite Element Method for Elliptic Problems, North-Holland, Amsterdam, 1978. MR0520174 (58:25001)

11. P.G. Ciarlet and P.-A. Raviart, The combined effect of curved boundaries and numerical integration in isoparametric finite element methods, The Mathematics Foundations of the Finite Element Method with Applications to Partial Differential Equations (A. K. Aziz ed.), Academic Press, Inc., 1972, pp. 409-474. MR0421108 (54:9113)

12. P. Clément, Approximation by finite element functions using local regularization, RAIRO., 9 (1975), 77-84. MR0400739 (53:4569)

13. J.G. Conlon and A. Naddaf, On homogenization of elliptic equations with random coefficients, Electron J. Probab., 5 (2000), 1-58. MR.1768843(2002j:35328)

14. L.J. Durlofsky, Numerical calculation of equivalent grid block permeability tensors for heterogeneous poros-media, Water. Resour. Res., 28 (1992), 699-708.

15. W. E, Homogenization of linear and nonlinear transport equations, Comm. Pure Appl. Math., 45 (1992), 301-326. MR1151269 (92k:35026)

16. W. E and B. Engquist, The heterogeneous multiscale methods, Comm. Math. Sci., 1(2003), 87-132. MR 1979846 (2004b:35019)

17. W. E and B. Engquist, The heterogeneous multiscale method for homogenization problems, submitted to MMS, 2002.

18. W. E and B. Engquist, Multiscale modeling and computation, Notice Amer. Math. Soc., 50 (2003), 1062-1070. MR2002752

19. W. E and X.Y. Yue, Heterogeneous multiscale method for locally self-similar problems, Comm. Math. Sci., 2 (2004), 137-144.

20. Y.R. Efendiev, T. Hou and X. Wu, Convergence of a nonconforming multiscale finite element method, SIAM J. Numer. Anal., 37 (2000), 888-910. MR1740386(2002a:65176)

21. M.I. Freidlin, Functional Integration and Partial Differential Equations, Princeton, NJ, 1985. MR0833742 (87g:60066) 
22. M.I. Freidlin and A.D. Wentzell, Random Perturbations of Dynamical Systems, second edition, Springer-Verlag, 1998. MR1652127 (99h:60128)

23. N. Fusco and G. Moscarielio, On the homogenization of quasilinear divergence structure operators, Ann. Mat. Pura. Appl., 146 (1987), 1-13. MR0916685 (89a:35025)

24. D. Gilbarg and N.S. Trudinger, Elliptic Partial Differential Equations of Second Order, second edition, Springer-Verlag, New York, 1983. MR0737190 (86c:35035)

25. T. Hou and X. Wu, A multiscale finite element method for elliptic problems in composite materials and porous media, J. Comput. Phys., 134 (1997), 169-189. MR.1455261(98e:73132)

26. I.G. Kevrekidis, C.W. Gear, J.M. Hyman, P.G. Kevrekidis, O. Runborg and C. Theodoropoulos, Equation-free, coarse-grained multiscale computation: enabling microscopic simulators to perform system-level analysis, Comm. Math. Sci., 1 (2003), 715-762. MR2041455

27. J. Knap and M. Ortiz, An analysis of the quasicontinuum method, J. Mech. Phys. Solids., 49 (2001), 1899-1923.

28. S.M. Kozlov, Homogenization of random operators, Math. USSR Sb., 37 (1980), 167-180. MR0542557 (81m:35142)

29. A.M. Matache, I. Babuska and C. Schwab, Generalized p-FEM in homogenization, Numer. Math., 86 (2000), 319-375. MR.1777492 (2001f:65139)

30. G.W. Milton, The Theory of Composites, Cambridge University Press, 2002. MR1899805 (2003d:74077)

31. P.B. Ming and X.Y. Yue, Numerical methods for multiscale elliptic problems, preprint, 2003.

32. S. Moskow and M. Vogelius, First order corrections to the homogenized eigenvalues of a periodic composite medium, Proc. Royal Soc. Edinburgh. Sec. A., 127 (1997), 1263-1299. MR1489436 (99g:35018)

33. F. Murat and L. Tartar, H-Convergence, Cours Peccot, 1977. Reprinted in Topics in the Mathematical Modeling of Composite Materials (A. Cherkaev and R. Kohn eds.), Birkhäuser, Boston, 1997, pp. 21-43. MR 1493039

34. G. Nguetseng, A general convergence result for a functional related to the theory of homogenization, SIAM J. Math. Anal., 20 (1989), 608-623. MF0990867(90j:35030)

35. J.T. Oden and K.S. Vemaganti, Estimation of local modeling error and goal-oriented adaptive modeling of heterogeneous materials. I. Error estimates and adaptive algorithms, J. Comput. Phys., 164 (2000), 22-47. MR1786241 (2001e:74079)

36. G.C. Papanicolaou and S.R. S. Varadhan, Boundary value problems with rapidly oscillating random coefficients, at Proceedings of the conference on Random Fields, Esztergom, Hungary, 1979, published in Seria Colloquia Mathematica Societatis Janos Bolyai, North-Holland, 27 (1981), pp. 835-873. MR0712714 (84k:58233)

37. R. Rannacher and R. Scott, Some optimal error estimates for piecewise linear finite element approximations, Math. Comp., 38 (1982), 437-445. MR0645661 (83e:65180)

38. C. Schwab and A.-M. Matache, Generalzied FEM for homogenization problems, Multiscale and Multiresolution Methods: Theory and Applications, Lecture Notes in Computational Sciences and Engineering, Vol. 20 (T. J. Barth, T. Chan and R. Heimes eds.), Springer-Verlag, Heidelberg, 2002, pp. 197-237. MR1928567|(2003i:65115)

39. R. Scott, Optimal $\mathrm{L}^{\infty}$ estimates for the finite element method on irregular meshes, Math. Comp., 30 (1976), 681-697. MR0436617 (55:9560)

40. S. Spagnolo, Convergence in energy for elliptic operators, Numerical Solutions of Partial Differential Equations-III (SYNSPADE 1975, College Park, MD, May 1975) (B. Hubbard ed.), Academic Press, New York, 1976, pp. 469-499. MR0477444 (57:16971)

41. L. Tartar, An introduction to the homogenization method in optimal design, Optimal shape Design (A. Cellina and A. Ornelas eds.), Lecture Notes in Mathematics, Vol. 1740. Springerverlag, 2000. pp. 47-156. MR.1804685

42. J. Xu, Two-grid discretization techniques for linear and nonlinear PDE, SIAM J. Numer. Anal., 33 (1996), 1759-1777. MF.1411848 (97i:65169)

43. V.V. Yurinskii, Averaging of symmetric diffusion in random media, Sibirsk. Mat. Zh. 23 (1982), no. 2, 176-188; English transl. in Siberian Math J., 27 (1986), 603-613. MR0867870 $(88 \mathrm{e}: 35190)$

44. V.V. Zhikov, On an extension of the method of two-scale convergence and its application, Sbornik: Mathematics, 191 (2000), 973-1014. MR1809928 (2001k:35026)

45. V.V. Zhikov, S.M. Kozlov and O.A. Oleinik, Homogenization of Differential Operators and Integral Functionals, Springer-Verlag, Heidelberg, 1994. MF 1329546 (96h:35003b) 
Department of Mathematics and PACM, Princeton University, Princeton, New JerSey 08544 and School of Mathematical Sciences, Peking University, Beijing 100871, PeoPLE'S REPUBLIC OF CHINA

E-mail address: weinan@Princeton.EDU

No. 55, Zhong-Guan-Cun East Road, Institute of Computational Mathematics, Academy of Mathematics and Systems Science, Chinese Academy of Sciences, Beijing 100080, People's Republic of China

E-mail address: mpb@lsec.cc.ac.cn

School of Mathematical Sciences, Peking University, Beijing 100871, People's RePUBLIC OF CHINA

E-mail address: pzhang@pku.edu.cn 